\title{
Trivium
}

Revue franco-allemande de sciences humaines et sociales - Deutsch-französische Zeitschrift für Geistesund Sozialwissenschaften

$14 \mid 2013$

Le Saint-Empire à l'époque moderne

\section{Le Saint-Empire moderne. Voie particulière et modèle pour l'Europe ou bien État de la nation allemande?}

\section{Georg Schmidt}

Traducteur : Pierre Monnet

\section{OpenEdition}

Journals

Édition électronique

URL : http://journals.openedition.org/trivium/4562

ISSN : 1963-1820

\section{Éditeur}

Les éditions de la Maison des sciences de l'Homme

Référence électronique

Georg Schmidt, « Le Saint-Empire moderne. Voie particulière et modèle pour l'Europe ou bien État de la nation allemande? », Trivium [En ligne], 14 | 2013, mis en ligne le 16 septembre 2013, consulté le 07 septembre 2020. URL : http://journals.openedition.org/trivium/4562

Ce document a été généré automatiquement le 7 septembre 2020.

\section{(c) $($ i) $(9)$}

Les contenus des la revue Trivium sont mis à disposition selon les termes de la Licence Creative Commons Attribution - Pas d'Utilisation Commerciale - Pas de Modification 4.0 International. 


\section{Le Saint-Empire moderne. Voie particulière et modèle pour l'Europe ou bien État de la nation allemande?}

\section{Georg Schmidt}

Traduction : Pierre Monnet

\section{NOTE DE L'ÉDITEUR}

Nous remercions M. Georg Schmidt ainsi que la maison d'édition Philipp von Zabern de nous avoir accordé l'autorisation de traduire ce texte pour le présent numéro. Wir danken Herrn Georg Schmidt und dem Verlag Philipp von Zabern für die freundliche Genehmigung, diesen Artikel in französischer Übersetzung zu publizieren.

1 Le fait de savoir si le Saint-Empire romain germanique constituait un État est, en soi, une question peu stimulante, la réponse dépendant qui plus est des représentations fondamentales que l'on se fait de l'État. La recherche allemande, obsédée par le modèle de l'État national souverain, s'est accordée à penser pendant près d'un siècle et demi et en dépit de toutes les ruptures institutionnelles que l'Empire ne formait pas un État. En référence à cette tradition, l'introduction du concept d' « Empire-État complémentaire » ${ }^{1}$ (« komplementärer Reichs-Staat ») ${ }^{*}$ a mis en émoi ${ }^{2}$ une partie de la communauté des historiens modernistes germanophones, tandis qu'une autre part accueillait avec sérénité ou bienveillance ce nouveau modèle interprétatif ${ }^{3}$. On pourrait ce faisant et en s'appuyant sur l'historicité de la formation de «l'État » procéder à l'analyse de l'Empire à partir de divers modèles. Mais une telle approche n'est pas sans conséquences sur l'appréciation de l'histoire allemande dans son ensemble. Définir l'Empire comme État et nation bouscule sensiblement le "grand récit " traditionnel : l'écart par rapport à une voie réputée normale de l'histoire européenne a jusqu'à présent conféré au passé 
allemand une signification pourvue d'une finalité tantôt légitimante tantôt déstructurante, mais toujours facteur d'intégration politique. Le concept d'Empire-État complémentaire ébranle l'idée de la singularité de l'histoire allemande moderne* sur un point capital, car il facilite la comparaison avec d'autres pays et oblige à considérer l'Allemagne comme partie prenante de l'Europe des États modernes. La notion d'Empire-État complémentaire ne peut dès lors servir ni de point de départ d'une « voie allemande particulière $\star^{4^{*}}$, ni d'archétype ou de modèle supra-étatique et supranational, ou d'équivalent fonctionnel de l'Europe contemporaine ${ }^{5}$.

2 La conception d'une étaticité complémentaire doit sa naissance à plusieurs facteurs. Parmi eux figurent :

1. Tout d'abord, le souci visiblement fort répandu de déterminer la nature institutionnelle complexe du "vieil Empire »* d'une manière plus précise que ne le permet le modèle courant du système politique. La construction plutôt incidente et récente de prédicats explicatifs tels que " système partiellement modernisé d'Empire ${ }^{6}$ ou « système refondu de pouvoir à deux étages ${ }^{7}$ parle en tout cas en ce sens. S'agit-il cependant d'expressions favorisant réellement la compréhension ou bien seulement de deux variantes incomplètes, tant de forme que de fond, d'un même malaise à saisir l'objet? Tandis que les approches (elles-mêmes disputées) relevant des théories de la modernisation ne signalent aucun changement significatif d'échelle, le «double étagement " semble pour sa part constituer une représentation impropre, dans sa simplicité, à rendre la complexité des structures constitutionnelles de l'Empire.

3 2. Ensuite, l'approche comparative qui traque les parallèles avec d'autres constructions étatiques européennes afin de pouvoir insérer l'Empire dans l'ordre européen des modèles évolutifs. En dernière analyse, chaque pays possède sa propre «voie particulière » d'avènement à l'État national souverain des XIXe et XXe siècles, fondé sur la trilogie réputée de longue durée et unissant les trois éléments toujours considérés comme unifiés d'un peuple, d'un territoire et d'un pouvoir organisé en monopole ${ }^{8}$.

4 3. Enfin la constatation suivant laquelle cette représentation de la construction étatique du XIXe siècle s'est elle-même historicisée entre temps, au point d'être aujourd'hui ajustée par les sciences politiques à une réalité en mutation'. Des représentations moins hermétiques de l'étaticité peuvent être néanmoins utilement convoquées à titre de variantes possibles afin d'ouvrir l'horizon historique à un modèle nouveau, à la fois globalisé et régionalisé, d'État ${ }^{10}$.

5 Compte tenu de la large palette des variations du concept d'État, il parait indéniable que la représentation d'un modèle fondé sur de nouvelles hypothèses puisse être appliquée à la compréhension du Saint-Empire moderne. La science politique actuelle ne renonce pas pour sa part au concept d'État au motif qu'il existe d'anciennes définitions provoquant d'autres associations d'idées, que Thomas Ellwein appelle des «barrières de compréhension $»^{11}$. Par contre, l'accent porté sur l'État national souverain comme seul étalon légitime pour mesurer le Saint-Empire moderne n'a pas seulement pour conséquence de faire réfléchir à partir d'un concept critiquable parce que devenu aujourd'hui une catégorie anachronique, mais aboutit aussi à se fonder sur des présupposés contestables à propos de l'accès réputé juste à l'État européen du XXIe siècle. Ce dernier est en effet un État coopératif ou corporatif, qui a transféré de larges portions de son pouvoir souverain de décision à des organisations supranationales (Union Européenne, ONU, OTAN), régionales (régions, communes, 
associations), voire à des comités d'initiative citoyenne. Mais l'État n'a pas disparu pour autant: il continue par exemple de garantir la légitimation démocratique et la sauvegarde des liens sociaux. En même temps, l'identité nationale ne cesse pas d'imprégner les représentations collectives de la fidélité et de l'appartenance, dans une proportion bien supérieure à celle que l'identité européenne peut assurer. L'État coopératif est un État formé d'une pluralité de composantes, inséré dans des systèmes culturels de références de grande comme de petite dimension, caractérisé par une "tendance à la pluralisation du pouvoir $»^{12}$. Ce concept nouvellement pensé d'un État ouvert au sommet comme à la base a sans doute plus à offrir en termes de prégnance génératrice de modèle et de capacité à la description que le concept, finalement demeuré vague, de système. Il semble bien indiqué pour restituer non seulement les réalités contemporaines, mais aussi - sous réserve d'un ajustement et d'un déplacement adapté aux conditions singulières de l'époque moderne - bien des caractéristiques structurelles du Saint-Empire ancien et plus largement de l'État européen prémoderne.

Que le concept d'État, qui dans ses variantes anciennes et plus récentes a été partout tenu en Europe pour suffisamment précis, complexe et ouvert afin de restituer terminologiquement les constructions modernes, de la monarchie absolue jusqu'à la République, doive rester à tout prix hors d'usage en Allemagne, pourrait bien être moins lié à des circonstances particulières qu'à la charge métaphysique intrinsèque dont il a spécialement imprégné la tradition scientifique allemande, au XIXe et encore tout au long du XXe siècle. L'État était son horizon moral, non seulement en qualité d'objectif à réaliser, mais aussi de pouvoir longtemps désiré et nationalement fêté sur le tard. C'est seulement dans cette perspective que le Saint-Empire des temps modernes a été ravalé au rang de conglomérat d'innombrables principautés, une situation qu'aujourd'hui encore les atlas historiques rendent à merveille. Même les expériences totalitaires restaient compatibles avec cet ancien modèle historique: la voie particulière d'un développement étatique allemand prétendument retardé, et à ce titre hautement agressif, a été convoquée pour expliquer et surmonter le désastre né de la dictature nazie et de la seconde guerre mondiale.

7 Cette construction continue encore d'agir en dépit de l'absence d'études capables de montrer empiriquement les différences de développement entre l'Allemagne et d'autres États tels que la France, l'Angleterre, les Pays-Bas ou la Pologne, dans la mise en œuvre de «l'État national moderne ». Seules de telles études pourraient objectiver cette comparaison et concrétiser la critique suivant laquelle le concept d'État susciterait dans le cas de l'Empire de fausses représentations. La preuve matérielle d'une carence des trois éléments fondateurs classiques de l'État dans le cas d'un Empire fédératif de nation allemande sera cependant bien difficile à administrer. Le pouvoir de « l'empereur et des états de l'Empire (ou de l'Empire tout seul)» ne s'étendait-il pas sur un territoire délimité ? Ne pouvait-on pas dénombrer à l'intérieur de leurs frontières les populations auprès desquelles ils pouvaient faire valoir leurs droits et leurs devoirs? Le pouvoir conjoint de l'empereur et de l'Empire n'était-il pas efficient? L'archétype du développement, qui continue toujours de voir dans l'État absolutiste du XVIIIe et du début du XIXe siècle "l'enfant modèle de la modernité ", selon les termes de Martin van Gelderen, l'indispensable stade intermédiaire et la condition nécessaire de l'État national souverain, doit être urgemment soumis à une « révision historique et idéologiquement critique ». Ni l'Angleterre ni les Pays-Bas n'auraient dû relever du processus de formation de l'État moderne fondé sur la typologie construite par Otto 
Hintze à partir du modèle prussien ${ }^{13}$, en raison de l'absence "d'une bureaucratie centralisée et d'une armée permanente $»^{14}$. Karin Friedrich, à l'exemple de la république nobiliaire polonaise, a même pu formuler l'hypothèse d'un passage direct d'un État prémoderne des états à « une monarchie constitutionnelle moderne ou à une république $»^{15}$. Des réflexions semblables peuvent être avancées tant dans le cas des Pays-Bas que de la Suisse ou de l'Empire-État complémentaire.

8 Dans la suite de ce propos, nous allons tenter d'expliquer pour quelle raison l'appréciation des contemporains, suivant laquelle l'Empire est bien l'équivalent de l'État allemand, a été abandonnée au XIXe siècle et comment la dépréciation qui en est résultée continue encore d'agir aujourd'hui. Puis on exposera sur cet arrière-plan en quatre points les avantages que présente le modèle explicatif de l'«Empire-État complémentaire ».

I.

9 L'évidence culturelle de l'Empire, qui s'exprime dans les propos tant spontanés que pesés des contemporains, imprégnait encore les projets de réforme et de constitution élaborés à la fin du XVIIIe siècle ${ }^{16}$. Ceux-ci entendaient certes en améliorer l'efficacité globale et étatique mais souhaitaient conserver en même temps les avantages de la constitution impériale et d'une compétence décentralisée de la décision. Les nouvelles avancées de la Révolution française en termes de citoyenneté et de liberté furent ainsi injectées dans l'ancien substrat constitutionnel ${ }^{17}$. Jusque dans les années 1790 , personne ne remit en doute que l'Empire, certes limité en puissance et peut-être même en efficacité, ne constituât l'État allemand en général. C'est à son correspondant français qu'on le comparait et, mesuré à cette aune, on lui prêtait une valeur supérieure en raison de la moindre intensité des mesures étatiques de répression, de ses espaces de liberté et de ses structures garantissant une sécurité à la fois juridique et économique, à l'exception notable de la pure hégémonie politique. Depuis le début du XVIIe siècle, la théorie de l'État de droit impérial (Reichsstaatsrechtslehre) a décrit sur plusieurs registres et souvent de manière idéalisée cet Empire et sa constitution, sans jamais lui refuser la qualité proprement étatique ${ }^{18}$. Tout au plus pouvait-on en discuter la forme étatique, entre monarchie, aristocratie ou constitution mixte. La formulation forgée et par la suite soustraite par Pufendorf lui-même d'un "quasi-monstre »* s'appliquait, en référence aux catégories aristotéliciennes, à un Empire qu'il caractérisait comme un système impérial et traitait à l'égal des autres États ${ }^{19}$.

10 Tandis que Johann Jacob Moser pouvait encore se satisfaire au XVIIIe siècle du précepte selon lequel "L'Allemagne se gouverne en allemand ${ }^{20}$, Johann Stephan Pütter proposait pour sa part une systématisation dont l'esprit conserve aujourd'hui encore toute son actualité : il appelait l'Empire un État constitué d'États ${ }^{21}$. Cette conception n'a pas seulement influencé la compréhension de l'État jusqu'à l'époque de la Confédération germanique mais a également constitué un modèle de pensée pour l'historiographie européenne plus récente ${ }^{22}$. Il fondait sa théorie sur l'exemple de l'Empire densifié de nation allemande né à la fin du Moyen Âge, sur un espace au sein duquel la paix perpétuelle avait été assurée par étapes successives ${ }^{23}$. À cette communauté de solidarité appartenaient, de Trente aux rivages de la Mer du Nord et de la Baltique, de la Poméranie à l'archevêché de Trèves ${ }^{24}$, les états, pays et habitants rassemblés en une "nation allemande » ou « Allemagne », que l'on pouvait mobiliser au 
service d'un Empire en train de se constituer en État ${ }^{25}$. L'empereur et (les états de) l'Empire, en qualité de représentants supérieurs de l'étaticité impériale et territoriale, constituaient un assemblage solidaire d'intervention - ce que les diètes impériales démontraient aux yeux de tous. En dépit de l'incontestable force étatique dont jouissaient les principautés ${ }^{26}$, les républiques urbaines $^{27}$ et les corps des états d'Empire ${ }^{28}$, l'Empire-État complémentaire disposait, avec la cour impériale ${ }^{29}$, les diètes impériales ${ }^{30}$ et la chambre impériale de justice ${ }^{31}$, de centres concrets; et la matricule d'Empire, les receveurs des deniers impériaux et les points de la collecte centrale de l'impôt impérial - souvent Francfort et Leipzig - lui conféraient un système fiscal doué d'un fonctionnement étonnamment efficace ${ }^{32}$. Avec l'aigle mono- et bicéphale, il disposait d'une symbolique omniprésente ${ }^{33}$; il pouvait mobiliser l'idée des libertés allemandes dans un combat tourné tant contre les adversaires intérieurs que les ennemis externes ${ }^{34}$. Il bénéficiait enfin, avantage non négligeable pour la solidité de l'ensemble, d'une poste impériale à l'organisation exemplaire ${ }^{35}$. L'empereur et l'Empire ont défendu conjointement la patrie allemande, en particulier contre les Turcs et les Français ${ }^{36}$, ont su trouver en 1555 à Augsbourg une solution novatrice aux conflits religieux $^{37}$, ont pu s'entendre en 1648 sur un nouvel ordre fondamental ${ }^{38} \mathrm{et}$, avec l'aide des cercles d'Empire, ont fait le nécessaire pour mettre en place une infrastructure fonctionnant somme toute de manière acceptable ${ }^{39}$ et, au XVIIIe siècle, une politique impériale - il est vrai au succès limité - face à la plus grande puissance militaire du temps ${ }^{40}$.

Les tribunaux d'Empire, dont l'activité a été longtemps sous-estimée, déployaient une action efficace, même en Poméranie, pourtant considérée comme loin du cœur de l'Empire ${ }^{41}$. Leur capacité à régler les conflits devint nécessaire dans toutes les parties de l'Empire afin de canaliser et, la plupart du temps, d'apaiser les querelles que le cadre territorial ne pouvait plus réguler. Cette constatation ne s'applique pas seulement, loin s'en faut, aux conflits entre sujets, déjà fort bien étudiés ${ }^{42}$, mais aussi et avant tout aux luttes opposant les puissants et les dominants entre eux, comme l'a récemment montré Siegrid Westphal à propos de l'introduction de la primogéniture, si prisée par l'ancienne littérature historique à cause de son efficacité étatique ${ }^{43}$. Chaque nouvelle recherche sur les cercles d'Empire, si longtemps sous-estimés, prouve au contraire le rôle capital qu'ils ont précisément tenu dans l'application des règlements généraux de l'Empire, dans la maîtrise des problèmes que posait à l'échelon suprarégional la question des infrastructures, de la bonne police et de la paix territoriale. Les études actuelles sur les actes des diètes d'Empire soulignent l'importance des assemblées impériales pour l'adoption en commun de normes contraignantes à l'échelle de toute l'Allemagne et pour la détermination d'une politique extérieure qui, au XVIIIe siècle, ne s'est pas contentée d'être simplement à la remorque de celle de l'Autriche ${ }^{44}$. Que les recès ou décisions des assemblées d'Empire ne soient pas restés simple lettre morte, rien ne le prouve mieux que la fidélité à payer les impôts impériaux ou ceux levés pour les campagnes contre les Turcs : c'est plus de $80 \%$ du montant fixé par la matricule d'Empire qui finit par rentrer, comme l'a démontré Winfried Schulze pour la fin du XVIe siècle ${ }^{45}$. Et il est secondaire, de ce point de vue, de savoir s'il convient d'expliquer cette discipline par une conscience forgée devant le danger commun ou par la crainte de mesures de coercition prises par l'Empire.

11 Au sein même de la nation allemande, souvent qualifiée (y compris dans les recès d'Empire du XVIe siècle) de patrie bien aimée ${ }^{46}$, se construit un réservoir de symboles partagés: l'idée d'Empire, l'aigle impériale, la Germania ou encore le personnage 
d'Arminius/Hermann ${ }^{47}$. D'importantes relations de clientélisme, soudées par les personnes ou le droit mais aussi par un connubium tissé au sein de la haute noblesse, ont tressé un réseau de liens formels et informels de dépendance et de parenté couvrant l'ensemble de l'espace allemand ${ }^{48}$. De même, la formation universitaire des élites politiques aux sources du ius publicum a-t-elle fondé une attitude communément partagée vis-à-vis des origines de l'Empire ${ }^{49}$. Les niveaux d'intervention si variés, le plus souvent décrits comme antagonistes ou concurrents les uns des autres, depuis les seigneuries d'Empire jusqu'aux espaces, systèmes et zones informels de l'économie, du clientélisme et de l'hégémonie, en passant par les solidarités de nature régionale, institutionnelle ou confessionnelle, ont tramé en raison même de leur recouvrement, un tissu d'interdépendances fonctionnelles qui, dans leur conjonction, ont fait apparaître l'Empire de nation allemande comme une entité délimitée au dehors et différenciée au-dedans. Les contemporains ont ainsi perçu cet échafaudage complexe et complémentaire de relations politiques, juridiques, économiques et culturelles comme une unité dans la diversité. L'abbé de Saint-Pierre, quoique mal interprété sur ce point, a même pu vanter cette construction comme un modèle utile à la création d'une future Europe fédérée ${ }^{50}$. La pluralité de centres diffusant chacun leur rayonnement national a pu ainsi être évaluée aussi positivement ${ }^{51}$ que les libertés allemandes dans toute leur diversité. Celles-ci étaient avant tout tournées contre la pensée monarchique ou absolutiste, mais pouvaient aussi porter des valeurs dépassant les intérêts des états et corps particuliers, telles que la garantie de la propriété, la tolérance religieuse et un certain libéralisme ${ }^{52}$.

En Allemagne, l'imprimerie à caractères mobiles n'a pas seulement influencé au plus haut point le mouvement réformateur, il a également déclenché une véritable révolution de la communication, parce qu'en raison de la juxtaposition de multiples censures, il se trouvait toujours des nouveautés que l'on ne pouvait interdire et qui valaient commercialement la peine d'être publiées. Le flot d'écrits occasionnels publiés comprenait des appels au respect de la dignité de l'ordre constitutionnel, des libertés allemandes, du système juridique ou même de sa propre langue et de la défense de l'Empire ${ }^{53}$, qui selon toute apparence transcendaient les intérêts qu'ils servaient. La diffusion massive des feuilles volantes, placards et journaux contribua, à côté de la Bible, du catéchisme luthérien et des innombrables traités d'édification religieuse, et par l'homogénéité de la langue allemande écrite qu'ils colportaient, à l'établissement de caractères, représentations et comportements d'allure "nationale ». Afin de se financer, la poste impériale, si l'on en croit Wolfgang Behringer, s'est ouverte dès l'origine aux usagers privés et rendit ainsi possible une intensification de la communication qui n'avait pas son pareil en Europe.

Même considéré sous ses aspects économiques, le prétendu éclatement [de l'Empire] n'est plus considéré depuis longtemps sous un angle négatif. Tandis que l'existence effective d'un système de finance et de crédit s'étendant à toute l'Allemagne n'est plus à démontrer ${ }^{54}$, non plus que les efforts nombreux pour créer un espace économique allemand $^{55}$, les recherches plus récentes soulignent combien la structure territoriale de l'Empire et la concurrence mutuelle ont augmenté l'esprit du risque et de l'innovation et, ce faisant, favorisé une introduction plus rapide des inventions et des procédés de production ${ }^{56}$. Tous ces indices plaident en faveur de l'existence d'un espace central de langue allemande à l'époque moderne, forgé par la constitution impériale, que l'on peut aisément comparer aux autres États européens eu égard aux cohésions culturelles et politiques qu'il offre. Partant de ce modèle interprétatif, l'écriture d'une histoire de 
l'Empire en tant qu'État de nation allemande vers 1800 a autant de sens que les synthèses similaires conçues pour les histoires nationales anglaise, danoise ou polonaise ${ }^{57}$.

14 À partir de la paix de Bâle de 1795, qui entraîna une partition de fait de l'Empire ${ }^{58}$, puis à la suite de la série de défaites éprouvées pendant les guerres de coalition et des dispositions fatales pour l'Empire des traités de paix conduisant à la perte des territoires situés sur la rive gauche du Rhin et enfin, en 1803, avec le changement profond imposé du dehors à la constitution de l'Empire ${ }^{59}$, germent dans certains milieux intellectuels de nouvelles conceptions d'une nation allemande dépouillée de son enveloppe impériale protectrice. Tandis que l'idée déjà ancienne d'une fédération d'États formant une "troisième Allemagne » débarrassée de l'Autriche et de la Prusse faisait de nouveaux émules ${ }^{60}$, Hegel et Schiller composaient les premiers fragments, dans un premier temps inédits, des Écrits constitutionnels (Verfassungsschrift) et de la Grandeur allemande (Deutsche Größe) ${ }^{61}$. Ces deux textes ont agité les sciences sociales allemandes tant sur la question de l'idée morale de l'État que sur la différence supposée entre nations étatiques et nations culturelles ${ }^{62}$. À tout prendre, ils sont avant tout l'expression manifeste de l'émergence vers 1800 chez les intellectuels allemands du cercle de Weimar-Iéna - citons encore Humboldt, Novalis et les frères Schlegel - d'une réflexion non seulement consacrée au Weltbürgertum, à l'Europe et à la chrétienté, mais aussi au lien unissant l'Empire, l'État et la Nation ${ }^{63}$. Différemment l'un de l'autre mais de manière tout aussi lourde d'avenir, Hegel et Schiller faisaient la distinction entre l'État et la Nation. L'un comme l'autre ne mettaient pas en exergue le substrat ethnique d'un "peuple», mais mettaient en avant une nation politiquement formée et insistaient sur l'indispensable éducation de sujets appelés à devenir des citoyens de l'État.

15 "L'Allemagne n'est plus un État ${ }^{64}$. Cette considération, devenue plus tard un véritable leitmotiv de la pensée de Hegel, identifiait - dans le droit fil de la tradition du XVIIIe siècle - l'Empire à l'Allemagne. Puisque, selon lui, l'État de la raison morale, censé ménager au citoyen la liberté la plus large possible, ne doit posséder qu'une instance centrale de décision et une administration fiscale en bon état de fonctionnement pour assurer des fonctions défensives et le cas échéant offensives, la qualité proprement étatique ne se définit dès lors et en dernier lieu que par sa capacité à mobiliser le pouvoir. L'État hégélien est un pur État de pouvoir, qui élève intellectuellement l'idée de souveraineté à des sommets jusqu'alors inconnus ${ }^{65}$. Puisque pour Hegel l'État porte intrinsèquement l'idée morale - entendons la liberté citoyenne, la forme constitutionnelle concrète qu'il peut revêtir lui importe peu. Il se situe de la sorte à l'origine d'une pensée de l'État devenue particulièrement prégnante en Allemagne, qui s'attache en premier lieu à concevoir le déploiement externe du pouvoir et, pour cette raison, croit pouvoir renoncer en cas de nécessité aux garanties constitutionnelles de la liberté.

Schiller au contraire distingue l'Empire encore existant et la nation en cours de formation politique et territoriale ${ }^{66}$. Il appuie sa réflexion sur une nation allemande anoblie par les arts et les sciences, dont il fait dériver le caractère exemplaire ainsi que la mission historique de «l'Allemand » sur terre. Son État ou condition de liberté n'est justement pas défini comme une liberté assignée de l'extérieur par un État de pouvoir mais échafaudée à partir d'un pouvoir législatif à tout le moins volontairement consenti par la majorité. À ses yeux, la nation allemande est constituée d'un certain 
nombre de libertés, de droits et de coutumes et, partant, d'une culture politique commune. Celle-ci lui semble désormais suffisamment assurée pour qu'elle ne menace pas de sombrer, même lorsque l'Empire qui lui assurait jusqu'alors la protection de la fédération fait naufrage. C'est uniquement parce qu'existe déjà une société nationale politiquement et territorialement formée que Schiller peut postuler l'existence d'une nation allemande affranchie des formes concrètes de l'État et du pouvoir. Cela ne signifiait en rien une nation culturelle sans liens ni attaches. Lorsque le fragment de Schiller parut, en 1871 seulement, il se trouva alors confronté à une réalité sociale qui ne put et ne voulut plus en retenir que la charge politique expansive. C'est qu'entretemps le modèle hégélien de l'État souverain de pouvoir s'était imposé.

On avait oublié que le Saint-Empire n'avait nullement disparu en 1806 sans bruit ni fracas, comme la recherche allemande a bien voulu le laisser croire encore récemment ${ }^{67}$. Fichte pouvait encore parler en 1808 avec le plus grand respect du « corps impérial » comme de la nation des États allemands. Extérieurement, l'État et la nation lui apparaissaient séparés l'un de l'autre, mais ils n'en étaient pas moins harmonieusement unis, de manière visible par l'Empire, et invisible " par une foule de coutumes et d'institutions ». Il délivre un cinglant démenti à l'idée qu'un seul État particulier ait dominé toute l'Allemagne :

«Aucun prince de naissance allemande n'a jamais pris sur lui de n'imposer pour patrie à ses sujets que le territoire délimité par les montagnes ou les fleuves soumis à son pouvoir [...] Une vérité qui ne pouvait être proclamée dans un endroit pouvait l'être dans un autre [...] et ainsi [...] régnait pourtant en Allemagne, si l'on considérait celle-ci comme un tout, la plus extrême liberté de recherche et d'échange qu'un peuple ait jamais connue ${ }^{*}$.

La différence entre État et Nation mérite certes considération, mais autrefois «les ressorts relevant de l'une et de l'autre " ne pouvaient «jamais entrer en conflit [...] L'amour supérieur de la patrie pour le peuple de nation allemande considéré dans son ensemble devait nécessairement former le principe conducteur supérieur au sein de chaque État allemand singulier [...] ». Le seul danger que l'on pût craindre résidait dans la tentative qu'entreprendrait un État allemand «d'unir sous sa domination la nation allemande tout entière ${ }^{68}$.

Suivant l'usage linguistique de l'époque moderne, Fichte n'introduisait pas de distinction véritable entre État et nation. L'Empire pour lui était la nation allemande ; seulement il avait disparu entre temps. C'est pourquoi il se fondait sur une langue commune à tous et à ce titre naturellement unifiante, érigée en socle d'une nation que ne séparaient plus les états ou les corps, pour peu que les États particuliers soient mus par le principe de l'amour national. Après la disparition de l'Empire, Fichte plaça plus qu'il ne le faisait auparavant le substrat ethnique et culturel au cœur du concept de nation. Puisqu'il concevait la nation comme tournée contre toutes les autres entités étatiques distinctes, il ménageait toutefois ouvertement la possibilité d'interprétations nationalistes et même étatiquement dominatrices ${ }^{69}$. Cependant, l'État qu'il concevait devait pouvoir se rendre lui-même superflu dans un environnement cosmopolite ${ }^{70}$.

19 À tort ou à raison, Fichte, Schleiermacher, Arndt ou le "père de la gymnastique " Jahn, en raison de l'importance qu'ils accordent à une nation comprise en un sens plus fortement culturel et ethnique et de leur revendication parallèle d'un État fort, font office d'« inventeurs » des théories modernes de l'État et de la nation et, partant, du nationalisme en Allemagne ${ }^{71}$. Après que le dualisme allemand et Napoléon eurent conduit en 1806 à la fin du Saint-Empire ${ }^{72}$, les conceptions métaphysiques de l'État ${ }^{73}$ et 
sacralisées de la nation (l'autel de la Patrie) ${ }^{74}$ provoquèrent non pas immédiatement mais sur le long terme une rupture significative dans la mémoire du passé étatique de l'Allemagne. Les expériences accumulées aux côtés de princes désormais réellement souverains ou au sein de petits États, qui ne visaient l'unité nationale ni en un sens culturel ni en un sens politique, amenèrent certains défenseurs de l'idée nationale issus des élites de l'Allemagne septentrionale et moyenne à trouver des attraits au modèle français, pourtant honni par l'État de puissance et la conception prussienne d'un rassemblement de sujets aussi uni et structuré que possible sous la forme d'un État centralement régi. Une « borussification » de l'histoire allemande n'en résulta pourtant pas encore.

20 L'idée d'un accomplissement prussien de l'État souverain allemand avait surgi au cours de la Guerre de Sept Ans ${ }^{75}$, mais n'avait enflammé au tout début que des poètes et penseurs prussiens, parce que l'obsession prêtée à Frédéric II - seuls ses sujets étaient dignes d'appartenir à une nation prusso-allemande - la faisait apparaître ailleurs comme une menace d'annexion. Après 1806 , cette idée ancienne n'était pas morte ${ }^{76}$. Tandis qu'en Allemagne du Sud, l'Empire pouvait encore servir à incarner une idée fédérative de l'État et de la nation ${ }^{77}$, on pouvait au Nord envisager de nouvelles options $^{78}$, en raison de ce que l'on y avait vécu pendant la Confédération du Rhin ${ }^{79}$ et la Confédération germanique ${ }^{80}$. Selon Fichte, qui en 1808 encore dénonçait avec force l'idée de la domination d'un seul État sur l'Allemagne, la Prusse était bel et bien devenue en 1813 un "État allemand", que son génie et son histoire pressaient de mettre "ses pas dans ceux de l'Empire». Dans sa quête d'un "champion de la germanité ", ses pensées se tournaient vers le roi de Prusse, qui devait dorénavant se placer selon lui à la tête de la patrie allemande et intégrer la Prusse dans le giron de l'Allemagne ${ }^{81}$. Barthold Georg Niebuhr pour sa part reconnaissait en 1814 l'existence d'une " véritable nation prussienne » au sein de la nation allemande. La Prusse était « la patrie commune de tout Allemand qui se distinguât dans les sciences, les armes et le gouvernement $"^{82}$. Il n'y avait là rien de nouveau, et pourtant, dans un contexte dramatiquement bouleversé, l'idée d'une unité allemande forgée par la Prusse et son système de valeurs ne résonnait plus comme un processus sécessionniste. De tels projets ne pouvaient toutefois pas encore se concrétiser: c'est bien en vain qu'on réclamait un État allemand souverain dans les universités de l'Allemagne moyenne ${ }^{83}$. Les nouvelles esquisses de la nation combinaient assurément des traditions fort nébuleuses: la prétendue extension territoriale de l'Empire médiéval, la nation de langue allemande et le droit présumé de chaque peuple ou de chaque nation à s'unir au sein d'un même État. C'est dans cette confusion hautement explosive d'ambitions ethniques, culturelles et politico-étatiques que surgit l'idée jusqu'alors inconnue d'un État grand-allemand, modelant un État national allemand sans considération des frontières existantes ou de sentiments d'appartenance ${ }^{84}$. L'idée d'une réunion de tous les Allemands au sein de l'Empire n'existait pas avant 1806. L'Empire-État complémentaire reposait sur des composantes politico-étatiques et non sur des assignations ethniques ou linguistiques.

21 À l'instar de Niebuhr, Humboldt et quelques autres, qui avaient formulé précocement le rôle moteur que la Prusse devait tenir dans l'intégration étatique de l'Allemagne, David Hansemann, natif d'Aix-la-Chapelle, adressait en 1830 au roi Frédéric Guillaume III un mémoire dans lequel il demandait que la Prusse se place à la tête de la "promotion de la liberté germanique ", parce que cela servait ses intérêts, à savoir "réunir les États allemands au sein d'une gerbe nouée en commun $»^{85}$. Mais à cette date il n'était pas 
encore établi si c'était à l'Allemagne d'absorber la Prusse - ainsi que le réclamaient Fichte en 1813 et le baron Charles Henri de Stein en 1812 - ou bien le contraire. Un historien comme Gustav Adolf Harald Stenzel, dans sa biographie de Frédéric II, pouvait certes suggérer en 1837 l'idée d'une mission prussienne à travers la thèse d'un rôle protecteur exercé par la Prusse sur l'Allemagne ${ }^{86}$, mais dans le même temps Johann Friedrich Böhmer voulait élever à Francfort un monument à la gloire du Saint-Empire romain. Ce dernier estimait encore au milieu du siècle qu'une nouvelle organisation de l'Allemagne ne pouvait se nourrir qu'aux sources de l'Empire et de son ancienne constitution unitaire.

«Ce n'est pas parce que ce lien a perdu de sa force au cours du temps qu'il ne nous en a pas signifié beaucoup [...] Jusqu'au dernier moment, c'est bien encore l'empereur, auquel les princes et le peuple avaient juré fidélité, qui demeurait le garant de l'unité face au dehors, le parrain de la représentation nationale par le biais des diètes d'Empire auxquelles siégeaient réunis en trois bancs les princesélecteurs, les princes et les villes, et enfin le comptable de la justice grâce aux tribunaux d'Empire $»^{87}$.

C'est seulement à partir du milieu du siècle que le Saint-Empire moderne ne fut plus considéré que par un petit nombre de spécialistes comme une structure politique de large rassemblement. Après la révolution allemande de 1848-1849, un processus de «borussification" s'empara de toute la science historique. Avec son Histoire de la politique prussienne, Gustav Droysen ${ }^{88}$ fournit sans doute aux " artisans historiens de la petite Allemagne » l'œuvre la plus décisive ${ }^{89}$. La mémoire culturelle dessinait désormais une image plutôt ambivalente de l'Empire disparu: ses prétendues force et grandeur furent élevées au rang de mythe exemplaire grâce à une opération de revalorisation du haut Moyen Âge. Le contraste fut souligné par la chute de 1806, qui ne provoquait souvent plus que des réactions de sarcasme et de moquerie. La fixation historiographique sur l'État national souverain, désiré puis peu après légitimé, aboutit à dénier au Saint-Empire moderne tout caractère étatique, à la différence du lointain État médiéval et des États princiers, au premier rang desquels la Prusse-Brandebourg, qui en avaient maintenu l'héritage. On peignit le tableau d'un Empire dont la décadence dramatique n'aurait pas cessé depuis les Staufen ${ }^{90}$, un Empire précipité dans sa chute par les opposants à la Réforme, par la Guerre de Trente Ans et par la paix de Westphalie. Dès le milieu du XIXe siècle, l'historiographie s'employa à faire du SaintEmpire moderne un conglomérat éclaté, incapable de se défendre et livré au contrôle des puissances étrangères, dont le seul mérite consistât à ne pas freiner la formation de l'État prussien ${ }^{91}$. Cette interprétation ne fut d'abord partagée que par les historiens allemands fixés sur la Prusse. L'antithèse " grand-allemande » insistait davantage pour sa part sur l'idéal de l'Empire médiéval, dans lequel elle cherchait des sources d'inspiration pour le présent ${ }^{92}$. Les deux tendances s'accordaient cependant sur le fait que l'Empire qu'elles avaient ainsi déconstruit ne correspondait plus aux nouvelles représentations d'un État souverain. Tout cela, qui plus est sous l'emprise permanente de l'image d'une Confédération germanique paralysée dans sa politique extérieure ${ }^{93}$, alimenta le reproche rétrospectivement adressé au Saint-Empire moderne de n'avoir pas su, contrairement à ses voisins, engendrer des structures étatiques aptes à créer une cohésion nécessaire. C'est la raison pour laquelle on lui contesta toute existence politique propre au sein du système étatique européen et on le tint en grande partie responsable de la misère présente de l'Allemagne.

Peu de temps après, l'Empire bismarckien, fort de ses évidents succès, parut bien confirmer cette hypothèse : Heinrich von Treitschke et les manuels scolaires devinrent 
les partisans les plus efficaces de l'ascension prusso-allemande ${ }^{94}$. La critique fondamentale formulée par Hermann Baumgarten ne put aller bien loin :

« Il [Treitschke] ne mesure tout ce qui se passe dans le monde qu'à l'aune d'un seul critère : est-ce bien avantageux pour la Prusse, est-ce bien l'aveu de reconnaissance de tous les mérites revenant à la Prusse pour avoir libéré l'Allemagne, est-ce bien la preuve que l'avenir de l'Allemagne passe par la Prusse ? $»^{95}$.

C'est à l'actif de Treitschke bien plus qu'à celui de Baumgarten que furent inscrites les représentations et sensibilités nationales de l'Empire bismarckien. Ses interprétations, ses légendes marquèrent des générations d'historiens. Friedrich Meinecke en 1907 reconnaissait dans les années 1800 "une singulière constellation en Allemagne [...] suivant laquelle les seuls véritables fondements d'un État national moderne reposaient non sur le sol de la nation allemande mais sur celui de l'État prussien ${ }^{96}$. Il sondait alors les origines du problème appelé "prusso-allemand » et examinait "les idées nationales à la lumière de leur adéquation à l'État prussien [...], et de leur compatibilité avec son autonomie et son génie ». Il inventa ce faisant la prétendue dichotomie entre « la nation de l'État prussien et la nation culturelle allemande qui voulait se faire nation de l'État allemand $»^{97}$. Ses thèses continuaient encore de sévir un siècle plus tard :

« Depuis le début du XIXe siècle, les esprits libéraux n'associaient plus à la vieille et impuissante Allemagne, pieusement censée servir aujourd'hui de modèle à la République fédérale, que les termes de trou provincial ${ }^{*}$, de petit-bourgeois, de bonnet de nuit, de philistin, de corruption $»^{98}$.

Volker Press le souligne bien : «Les penchants des historiens ont de tout temps été mus par les représentations et les aspirations de leur propre époque ${ }^{99}$. Dans l'Empire wilhelminien, l'histoire de l'Allemagne moderne se résumait à la glorieuse Réformation luthérienne contre Rome et les catholiques, accusés d'avoir empêché la réalisation de l'unité nationale sous l'égide des Protestants. La déliquescence intérieure puis la Guerre de Trente Ans en étaient la conséquence logique, et les puissances européennes pouvaient sans entraves régler leurs conflits sur le sol allemand. Seules l'ascension de la Prusse et les Lumières, dans lesquelles se profilait l'affirmation d'un nouveau sentiment national, annonçaient des temps meilleurs ${ }^{100}$. L'Allemagne étatiquement éclatée et contrôlée par les puissances extérieures était distancée par les autres États européens ou du moins avait confié les rênes à la Prusse et à l'Autriche.

"L'idée selon laquelle l'État soudé par le pouvoir et l'institution était la seule force motrice de l'histoire européenne et que l'Allemagne dans son ensemble présentait de ce point de vue un déficit face aux États centralisés d'Europe de l'Ouest, et que la voie d'accès à l'État moderne avait été empruntée ici non par l'Empire mais par les principautés territoriales, demeure finalement jusqu'aujourd'hui largement partagée ${ }^{101}$

Volker Press, à travers d'innombrables études, a pourtant œuvré comme aucun autre historien à la révision d'une telle image. En cela il pouvait s'appuyer à la fin des années 1950, dans le contexte d'une prise de distance vis-à-vis de la catastrophe de l'État de pouvoir allemand, sur les travaux consacrés au Saint-Empire moderne par Heinrich Lutz $^{102}$, Gerhard Oestreich ${ }^{103}$ et avant tout par Friedrich Hermann Schubert ${ }^{104}$ et Karl Otmar Freiherr von Aretin ${ }^{105}$. Ils avaient commencé à réinterpréter ce que l'ancienne historiographie avait identifié comme des déficits étatiques et structurels caractéristiques de l'Empire, pour les inscrire au bénéfice d'un contexte non étatique et supranational situé au cœur même de l'Europe moderne ${ }^{106}$.

Fondée sur le modèle heuristique de «l'Empire comme système politique " emprunté aux sciences sociales systématiques* et développé à travers la coopération stimulante 
établie entre Volker Press et Peter Moraw, une nouvelle phase de la recherche consacrée au Saint-Empire put s'épanouir, couronnée de résultats extrêmement fructueux ${ }^{107}$. On put enfin démontrer quelles fonctions capitales et tellement indispensables aux États princiers eux-mêmes cet Empire a pu remplir jusque vers 1800. Au cours des 25 dernières années, toute une série de nouvelles études, bien éloignées de toutes les interprétations encore marquées par l'approche de l'État souverain, ont spécifiquement défini le système impérial comme une organisation politique, certes plus ou moins habile à se défendre à l'extérieur, mais capable à tout le moins, en tant que construction constitutionnelle et juridique, d'assurer une cohésion interne aux différents États allemands et de ménager des espaces de liberté s'opposant aux tendances absolutistes des princes ${ }^{108}$. Wolfgang Reinhard est même allé plus loin en qualifiant l'Empire de "premier État constitutionnel» au regard des règlements constitutionnels négociés pendant la Guerre de Trente Ans au détriment du pouvoir monarchique ${ }^{109}$. Il suivait ainsi Johannes Burkhardt qui, la même année, définissait la paix de Westphalie comme une "constitution générale de l'État à l'époque moderne " ${ }^{110}$.

Entre temps, le nombre sans cesse croissant d'études et de recherches sur l'Empire ont conduit le concept de "système" comme catégorie explicative aux limites de son emploi. Les historiens d'aujourd'hui constatent ce qu'en son temps Samuel Pufendorf remarquait déjà, à savoir que le concept de système ne résout pas les problèmes mais les déplace à un autre niveau ${ }^{111}$. Afin de mieux rendre justice terminologiquement parlant à cet Empire de nation allemande que la recherche récente avait si bien " étoffé »112, la catégorie d'Empire-État complémentaire a pu être proposée, forgée à partir d'un prédicat interprétatif et de concepts puisés dans la documentation ellemême ${ }^{113}$. Par ce biais d'un renvoi de principe au caractère étatique, on a voulu indiquer qu'il s'agissait là d'une formation étatique surgie d'un Empire dont l'organisation féodale n'a pas débouché sur la création d'un État souverain absolu ${ }^{114}$. L'adjectif " complémentaire » sous-entend que ce que l'on comprend communément comme une instance étatique unique et qui en France était concentrée dans les mains du roi ou était décrite en Angleterre comme "le roi en son parlement", se trouvait dans l'Allemagne de l'époque moderne partagée entre plusieurs instances. L'empereur, constitutionnellement circonscrit en qualité de monarque, réglait les affaires courantes du gouvernement, exerçait son droit de réserve, décidait, idéalement en concertation avec les états de l'Empire, de la guerre et de la paix et adoptait des règlements généraux dont la validité s'étendait à tout l'Empire. Les cercles d'Empire et les ligues garantissaient la paix territoriale et appliquaient des dispositions touchant les infrastructures et les règlementations de l'ordre social et économique, tandis que les États princiers et les villes assuraient de manière autonome une administration aussi efficace que possible et, en accord avec leurs sujets, imposaient une discipline sociale caractéristique de l'époque moderne. La notion d'«Empire-État complémentaire » semble suffisamment ouverte pour rendre compte d'évolutions très diverses, sans postuler pour autant le principe d'une unicité de développement de l'Allemagne susceptible d'interdire la comparaison pratique avec d'autres pays. 
II.

l'espace public prussien depuis la Guerre de Sept Ans puis enracinée au cours de la seconde moitié du XIXe siècle, n'a pas seulement produit la déclassification de cette structure historique mais a aussi imprimé sa marque au "grand récit » de l'histoire allemande jusqu'à nos jours. L'«invention" d'une voie séparée d'accès à l'État moderne, formulée vers 1900 de manière téléologique sur l'arrière-plan d'une marginalisation du Saint-Empire moderne puis conçue après 1945 dans un double sens explicatif et exonérant, est devenue une source de légitimation pour une Allemagne qui a reconnu sa faute et a clairement posé les jalons tant actuels qu'historiques de son destin: une démocratisation cohérente, un arrimage à l'Ouest et une intégration européenne opérée à distance critique de son propre État national. La figure prégnante d'une histoire de la décadence allemande au temps du Saint-Empire moderne demeure l'étape préparatoire nécessaire à tout récit d'un Sonderweg allemand: «L'Allemagne était bien, quoi qu'on en dise, un État et une nation en retard au XIXe siècle, qui voulurent à pas économiques et politiques forcés rattraper l'écart que l'Empire n'avait pu combler en raison de son étaticité déficiente [...] $»^{115}$. La faute en incombe finalement aux erreurs d'aiguillage des années 1500-1800 : «Au commencement était l'Empire ». Telle est l'origine du Sonderweg allemand ${ }^{116}$.

Tandis que cette historiographie rend ainsi le Saint-Empire moderne responsable de ces prétendus retards ou malformations, elle en vante en même temps les mérites en qualité d'exemple ou d'« équivalent fonctionnel » de l'Europe d'aujourd'hui. Il s'agit là d'une tournure bien discutable, car elle attribue à l'histoire prémoderne de l'Allemagne la fonction agissante d'une lointaine préhistoire de l'État totalitaire. Cette conception fonctionnaliste et moralisante n'est pas illégitime, mais comporte bien des risques: rétrospectivement, le Saint-Empire moderne perd de la sorte son identité et sa rationalité d'action propres et n'est plus que la variable d'un dogme anhistorique et didactique. Cette perspective, à suivre Joachim Whaley,

« échoue à reconnaître pleinement les voies par lesquelles l'Empire était le produit d'une expérience nationale allemande unique [...] Les pays allemands ne peuvent être simplement l'équivalent de l'Europe ; leur histoire ne peut tout bonnement pas être confondue avec celle de l'Europe. À l'instar d'autres parties de l'Europe, à l'Est comme à l'Ouest, ils possèdent leur propre histoire nationale ou ethnique; et l'histoire européenne, comme le présent (et l'avenir) de l'Europe, ne pourra assurément jamais être plus que la somme de ces différentes parties $»^{117}$.

La thèse du Sonderweg n'a pu convaincre que ceux qui voulaient assigner à l'Allemagne, pour des raisons politiques actuelles, une évolution spécifique. Qu'ils aient souhaité sur cette base construire une tradition «anti-occidentale» ou l'ériger en condition nécessaire à l'expérience criminelle du régime nazi, ses partisans l'ont toujours évaluée à la mesure d'un processus réputé normal d'évolution en Europe de l'Ouest, au terme duquel se trouvait l'État national souverain, moderne et plus ou moins démocratiquement légitime. Mais comme on l'a déjà mentionné, une vaste synthèse des écarts de l'histoire moderne allemande par rapport à cet idéal-type n'a jamais encore été réalisée. Jamais par exemple on n'a pu empiriquement prouver les prétendues dispositions des Allemands à l'autorité, attribuées à Luther et au luthéranisme, coupables d'avoir engendré une mentalité de la soumission et de l'obéissance au droit au détriment de la société civile, d'une culture de la résistance et d'un esprit 
démocratique. C'est pourtant du luthéranisme allemand que sont issues les idées des Monarchomaques, aide précieuse à l'établissement d'une tradition européenne de la résistance ${ }^{118}$. De même, les sujets de l'Allemagne du Saint-Empire moderne purent faire valoir leurs droits, de manière moins violente toutefois, parce qu'ils disposaient (en raison de l'étaticité complémentaire de voies juridiques autonomes) de recours que ne possédaient pas les autres pays. L'homme du commun en Allemagne n'était nullement un simple destinataire d'ordres, mais a pu faire usage des possibilités de participation qui lui étaient offertes au niveau local et régional pour négocier et imposer ses conceptions d'une société pacifiée et disciplinée ${ }^{119}$.

Les racines de la formation prétendument différée de l'État et de la nation allemands, un retard qui, dit-on, a conduit à une véritable hypostasie de l'État comme idée morale, à une confiance placée dans sa capacité juste d'action et à une attitude particulièrement agressive à l'égard des ennemis intérieurs et extérieurs, remontentelles réellement au Saint-Empire? Même pour la France du XVIIIe siècle, l'idée d'un État souverain, piloté par une autorité absolue et dont les décisions étaient absolument contraignantes à l'interne et ne pouvaient se voir opposer nulle résistance, à l'intérieur comme au dehors des frontières, tenait davantage de l'horizon d'attente que de la réalité établie ${ }^{120}$. Les relations internationales ont fonctionné de 1815 jusqu'au milieu du XXe siècle suivant cette représentation idéale. Dans le monde contemporain, devenu bien plus complexe quoique toujours structuré de manière étatique, le concept de souveraineté n'est tout au plus employé que comme la réminiscence historique d'un système dans lequel personne n'est en droit de remettre en cause l'autorité étatique de l'autre dans les limites de son territoire, ni de s'immiscer dans ses affaires intérieures.

Les historiens modernistes allemands et autrichiens ont appliqué le changement décisif de compréhension du concept d'État sur des territoires particuliers de l'Empire ${ }^{121}$ au plus grand profit de la recherche, mais ils demeurent souvent encore tributaires d'une conception ancienne selon laquelle les fondements de l'État moderne en Allemagne n'ont été jetés que dans un cadre particulier. À l'inverse, dans des pays comme la France, la Grande-Bretagne, l'Espagne ou les Pays-Bas, la mutation historiographique qui concerne la légitimation de l'État, portée par les concepts d'« État composite », de " monarchie mixte » ou de "royauté multiple »" connaît son plein accomplissement. Il apparaît ainsi que, même dans la France de l'époque moderne, où la souveraineté se concentrait dans la personne du roi comme nulle part ailleurs, se développaient des tendances étatiques indépendamment $\mathrm{du}$ monarque $^{122}$. Ces mises à jour historiographiques ne doivent pas amener à la simple adoption des interprétations françaises, espagnoles ou néerlandaises de l'État moderne, mais conduire à des réajustements. Cela peut-il se faire sans influer en retour sur un Empire à propos duquel ces modèles explicatifs avaient été autrefois formulés?

III.

31 La définition couramment proposée par l'historiographie allemande d'un Empire comme territoire supranational et supra-étatique de la fidélité jurée nourrit un recours au passé à des fins politiques actuelles: c'est sur cette base que la voie d'un avenir «meilleur » est tracée. Les reconstructions téléologiques sont toujours discutables en histoire, et dans le cas présent elles ne sont même pas politiquement justifiables, car bien des chemins conduisent à une Europe unie, une Europe des États-Nations 
constituant actuellement la variante la plus plausible ${ }^{123}$. À quoi s'ajoute, mais cela aussi doit être pris en compte lorsqu'il s'agit de réévaluer scientifiquement l'histoire allemande, le fait que les peurs suscitées chez nos voisins par les expériences antérieures reposent actuellement moins sur l'appréciation du passé national et étatique de l'Allemagne que sur l'ambition qu'on lui prête de vouloir jouer un rôle moteur en Europe. L'image qu'on leur présente dès lors d'un Empire supranational servant d'exemple à l'Europe ne contribue certainement pas à réduire ces préjugés.

En 1946, Elisabeth Schmittmann dans la revue Neues Abendland préférait pour l'Allemagne le modèle de l'Empire médiéval à celui de l'État national de 1871, parce qu'il lui semblait le garant d'un mélange réussi entre diversité et unité et d'une intégration pacifique dans la communauté internationale ${ }^{124}$. En réaction à de telles réflexions, l'idée d'Empire a aussitôt été présentée comme la racine de toute la souffrance causée par les Allemands à travers le monde. Friedrich Gaupp, avec l'approbation de la Direction de l'Éducation Publique, publia ainsi en 1948 son traité intitulé La falsification de l'idée d'Empire en Occident. Il ne voulait rien moins que la destruction de l'idée impériale "après qu'elle eut, à la fois comme concept et comme vision du monde, déterminé de manière si funeste et pendant des siècles le destin de l'Europe et, au-delà, de la civilisation tout entière ». Le terme même d'«Empire " incarne a priori « une revendication dominatrice sur les autres peuples». L'idée d'Empire, à le suivre, a de tous temps été " prétexte à dominer, et pas seulement le continent ", " et elle a toujours été considérée comme une ambition morale, comme un appel à l'extension au niveau international d'une culture spécifiquement nationale ». Le plus grand mensonge de l'histoire européenne a cependant consisté à affirmer que "l'idée continentale d'Empire était une idée allemande, destinée à légitimer la revendication dominatrice des Allemands sur les autres peuples et sur les autres races ». La notion d'Empire pour cette raison ne devait survivre d'aucune manière, "même pas en tant que rêve romantique, ni même en qualité de théorie pourvue de quelque pensée ethnique, religieuse ou supranationale de l'humanité $»^{125}$.

Gaupp et son traité sont depuis longtemps tombés dans l'oubli, et l'Empire allemand n'est plus. Pourtant en 2000, le Ministre de l'Intérieur français de l'époque, Jean-Pierre Chevènement, avançait des idées assez comparables. Il estimait que les Allemands rêvaient toujours du Saint-Empire romain germanique et entendait ainsi esquisser les contours d'une domination allemande : en tant que nation la plus importante d'Europe, l'Allemagne demandait la fin des États-nations pour mieux la dominer, comme aux temps du Saint-Empire ${ }^{126}$.

On peut comprendre ce jugement si l'on considère la dimension effrayante qu'évoque le souvenir d'un Empire multinational courant de la Toscane à la Mer du Nord et à la Baltique, de la Savoie au comté de Flandres, de la Slovénie et de la Silésie jusqu'aux confins des pays baltes. Même si toutes ces régions restaient apparemment en paix, elles n'en vivaient pas moins sous la férule allemande. Les territoires vassaux de l'Empire médiéval, et l'Empire idéal qui dépassait de loin ces frontières de la suzeraineté, englobaient des pays qui abritaient d'autres États à l'époque moderne. Des pans entiers de la Bourgogne relevaient de la France ou des Provinces Unies. Quel lien a bien pu représenter l'Empire pour eux ? L'« eurocompatibilité » de ces représentations, même étayée d'un rappel du cosmopolitisme propagé par l'Allemagne et de son engagement en faveur de l'intégration européenne, est loin d'être assurée. La question politique qui se pose n'est pas de savoir si l'histoire racontée ici est allemande ou 
centre-européenne, mais comment elle est reçue et quels sont ses effets ailleurs. L'Empire présenté comme supranational n'a-t-il pas encore toutes les raisons de continuer à résonner pour un Français, un Britannique, un Tchèque ou d'autres comme une revendication implicite à la domination? Que se passe-t-il pour un Français ou un Italien, quand il voit que ses traditions étatiques sont rejetées par les Allemands et transformées en argument politique dans le contexte de leur projet européen actuel? Des malentendus ne sont-ils pas déjà programmés?

De façon différente, il semble possible d'écrire un récit de l'histoire allemande strictement limitée pour l'époque moderne à l'espace de l'Empire-État, auquel appartenaient aussi les principautés héréditaires de l'Autriche. De la sorte, les anciennes mises en relation de questions et d'intérêts relevant de l'historiographie des XIXe et XXe siècles touchant l'État national ne seraient pas réactivées ${ }^{127}$, pas plus que ne "serait ressuscitée l'identité historique ranimée sur plusieurs siècles d'un État national des Allemands [sic!] $»^{128}$. Ces deux citations renvoient à une pensée en catégories qui doivent être rejetées d'une histoire nationale moderne à vocation européenne.

Le concept heuristique d'«Empire-État complémentaire » présente, à l'encontre de la conception d'un système impérial d'une fidélité féodale supranationale et supraétatique, quatre avantages.

1. Le Saint-Empire moderne, saturé par l'épaisseur de ses États, s'en trouve sans ambiguïté spatialement corrélé à la nation allemande constituée politiquement et territorialement, condition nécessaire à la comparaison avec d'autres pays.

Il existe en vérité plusieurs Saint-Empires. Lorsque les contemporains parlaient, dans les divers sens de la souveraineté politique ${ }^{129}$, du "Saint-Empire romain germanique ", de l'«Empire allemand», de l'«Empire et nation allemande », de l'« Empire notre patrie bienaimée » ou tout simplement de l'« Empire » ou de l'«Allemagne », ils ne désignaient pas autre chose que l'Empire plus ou moins institutionnalisé formé autour de 1500 et refondé après 1648 en "État constitutionnel ». Il s'agissait bien de l'œuvre produite, consciemment ou non, en commun par l'empereur et les états d'Empire tout au long du demi-siècle suivant 1495 , une structure conçue en réaction tant aux processus de construction de l'État observés chez les voisins qu'aux évolutions internes à l'Empire même. Cet Empire-État complémentaire fut d'abord une entreprise portée par l'Allemagne du Sud avec quelques extensions en Allemagne du Nord, mais finit depuis le milieu du XVIe siècle par s'étendre à tous les territoires compris entre le SudTyrol et la Mer du Nord et la Baltique d'une part, le Rhin Supérieur et l'archi-électorat de Trèves et la Poméranie de l'autre. Jusqu'à la Révolution française, le ressort de l'Empire-État complémentaire ne connut des changements que sur les marges du Rhin Supérieur. Il reposait sur trois épicentres : la cour impériale, la diète impériale, le tribunal de la Chambre impériale. Quelques lieux centraux subalternes possédaient également une dimension suprarégionale, comme les centres de l'administration des cercles d'Empire, la résidence de l'archichancelier impérial, les points de la collecte centrale de l'impôt impérial et les universités dans lesquelles était enseignée la théorie du droit de l'État impérial. C'est bien à partir de ces faits que peut s'écrire, comme pour d'autres pays, une histoire de l'Allemagne moderne ${ }^{130}$, et non pas à partir d'une féodalité imaginaire incluant les royaumes d'Italie et de Bourgogne ${ }^{131}$, d'une tout aussi chimérique nation culturelle et linguistique, ou encore d'une multitude d'états 
particuliers évoluant comme des électrons libres, et cela afin de déterminer justement les différences par rapport à d'autres États ou nations d'Europe.

2. Les spécificités de la formation moderne d'un État allemand sont terminologiquement éclairées par l'ajout du terme « Empire » et du corrélat explicatif « complémentaire ».

La notion d'Empire-État reliés par un trait d'union forme une catégorie relevant d'un progrès évolutif. L'Empire dans ses territoires allemands ne cesse de vouloir devenir davantage encore un État. La diète d'Empire de Worms en 1495, la première Réformation avec ses diètes sans empereur, le système d'organisation créé par la paix d'Augsbourg de 1555, la paix de Westphalie érigée en véritable loi fondamentale, les capitulations de l'élection impériale et l'approbation de la Pragmatique Sanction constituent autant d'étapes d'un tel processus. Cet Empire-État est dit " complémentaire " parce que l'on faisait appel aux institutions impériales lorsque les problèmes ne pouvaient être réglés au niveau inférieur. Mais l'inverse est également vrai puisque les cercles et états d'Empire pouvaient à leur tour être sollicités dès lors que le couple " empereur et Empire » ne parvenait pas à un accord. L'efficacité peu à peu constituée au cours des diètes impériales par le binôme formé de «l'empereur et des états d'Empire » a pu se substituer à la déficience étatique, non seulement des petits mais aussi d'un bon nombre de plus grands États territoriaux. En fin de compte, ni l'Autriche ni la Prusse ne pouvaient exister au XVIIIe siècle sans l'empereur ni l'Empire. La complémentarité correspond ainsi à la triple fonction d'une autonomie interne et étendue des pouvoirs corporatifs de l'Empire, de la formation de confédérations régionales, corporatives ou confessionnelles pour régler des problèmes généraux, et de compétences de contrôle et de vérification confiées aux institutions d'Empire, de manière à garantir aux habitants de cet Empire-État un certain degré de sécurité juridique et de liberté.

39 3. Les représentations ethniques de la nation restent étrangères au concept d'« Empire-État complémentaire».

Les représentations ethniques de la nation constituent à l'époque moderne le substrat culturel, ancré sur le recours par les humanistes aux valeurs et vertus attribuées par Tacite aux Germains, d'une construction allemande de la nation par la langue, l'origine et la culture. Cette nation allemande est certes corrélée à l'Empire-État, mais en dépasse de beaucoup et en multiples points les limites. On y trouve en effet aussi bien et à la fois le modèle emprunté à la France et la distance qui l'en sépare, l'attention accordée à un prétendu bien commun et aux intérêts économiques supérieurs prêtés à l'Empire-État, comme en témoignent les sociétés linguistiques ou les critiques «à la mode "* du XVIIe siècle, de même que les débats " nationaux ", théâtraux et littéraires du XVIIIe siècle. Dans le périmètre des frontières de l'Empire-État résidaient de surcroît des habitants qui ne se définissaient en rien par des attributs ethniques et culturels relevant de la nation allemande. Mais ce n'était pas différent dans d'autres pays. Quant aux populations établies en dehors des frontières de l'Empire-État complémentaire et qui se définissaient comme « allemandes » en raison de leur langue, de leurs origines ou de leurs traditions culturelles, elles n'ont pas vocation à être saisies par le concept d'Empire-État. Tel est le cas des Suisses allemands. Quand elles postulaient, jusque dans un XIXe siècle parfois fort avancé, une appartenance à la nation allemande, elles le comprenaient dans un sens ethnique et culturel qui ne sous-entendait pas un rattachement à l'Empire-État. 
4. L'Empire-État complémentaire fut une formation historique, d'une durée étonnamment longue et de nature à marquer l'histoire européenne.

«L'Empire était exclusivement allemand et il n'a jamais été démontré que ses structures fédérales étaient transposables. Il a inscrit des pertes territoriales avec la sécession de la Confédération helvétique et des Pays-Bas, mais n'a jamais enregistré de gains territoriaux. Il composait bien une partie de l'Europe, mais n'en a jamais constitué le microcosme. Son histoire ne fut que l'une des nombreuses histoires parallèles de ce qui demeura et continue de devoir être considéré comme une Europe des nations $»^{132}$.

Le Saint-Empire moderne présentait, en tant que nation et État des Allemands, un potentiel de développement de nature libérale digne de mémoire, qui tomba dans l'oubli dès que l'État national souverain commença à marginaliser tout le reste. Il serait bon de réinscrire dans la mémoire culturelle des Allemands les traditions fédératives, les ripostes antimonarchiques, les conceptions d'une certaine sécurité du droit et de la propriété issues de la tradition des libertés allemandes et fermement transcrites en 1648, ainsi que les acquis relativement importants d'une liberté de presse et de conscience dont jouissaient les sujets-citoyens, et de rappeler leurs valeurs historiques, afin de les réintroduire en tant que telles dans une culture mémorielle de l'Europe.

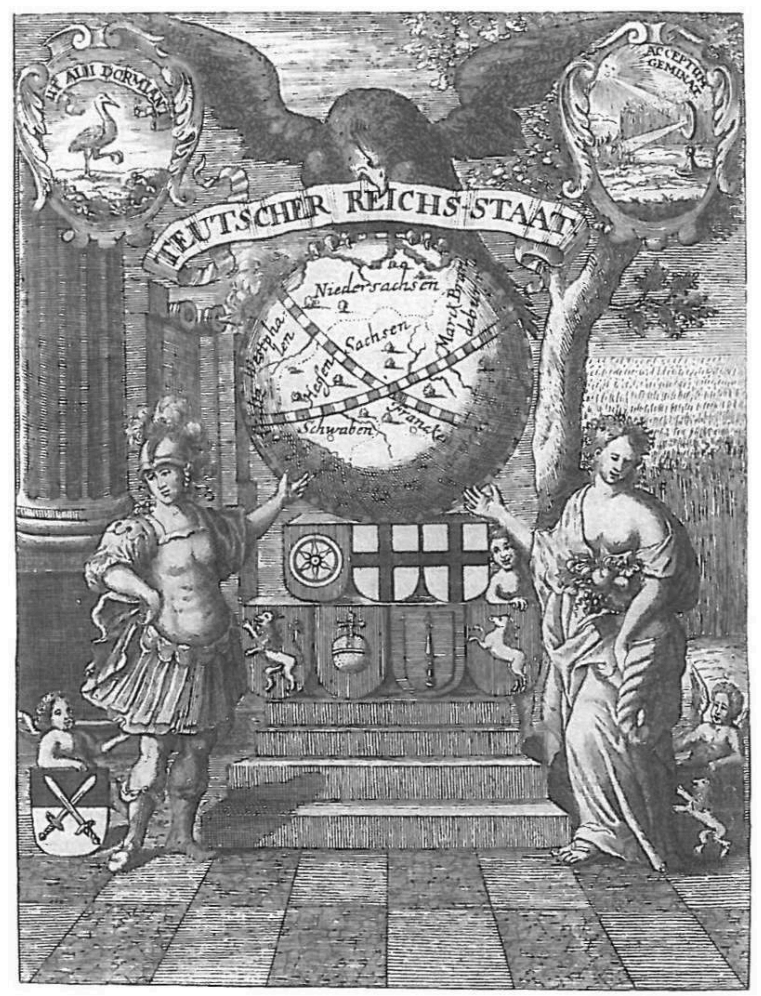

Johannes Sylverius Germanus [i. e. Christian Heinrich Krebs], Teutscher Reichs-Staat oder: Ausführliche und umständliche Beschreibung des Heil. Römisch. Reichs Teutscher Nation [...], II. Tomus, [...] vermehret durch Christoph. Laurent. Bilderbeck, Leipzig/Frankfurt a. M.: Hieronymius Friedrich Hoffmann, 1709, gravure illustrant la page de titre. 


\section{BIBLIOGRAPHIE}

Althoff, G. (2000) : « Das Mittelalterbild der Deutschen vor und nach 1945. Eine Skizze », in : Heinig, P.-J. [et al.] (dir.) : Reich, Regionen und Europa in Mittelalter und Neuzeit. Festschrift für Peter Moraw, Berlin, p. 731-749.

Angermeier, H. (1991) : « Deutschland zwischen Reichstradition und Nationalstaat. Verfassungspolitische Konzeptionen und nationales Denken zwischen 1801 und 1815 », in : Id. : Das alte Reich in der deutschen Geschichte, Munich, p. 449-521.

Aretin, K. O. Freiherr von (1967) : Heiliges Römisches Reich 1776-1806. Reichsverfassung und Staatssouveränität, vol. 1-2, Wiesbaden.

Aretin, K. O. Freiherr von (1988) : « Die Großmächte und das Klientelsystem im Reich am Ende des 18. Jahrhunderts », in : Maczak, A. (dir.) : Klientelsysteme im Europa der Frühen Neuzeit, Munich, p. 63-82.

Aretin, K. O. Freiherr von (1989) : « Das Heilige Römische Reich im Konzert der europäischen Mächte im 17. und 18. Jahrhundert », in : Schmidt, G. : Stände und Gesellschaft im Alten Reich, Stuttgart, p. 81-91.

Aretin, K. O. Freiherr von (1993-2000) : Das Alte Reich 1648-1806, vol. 1-3, Stuttgart.

Arndt, J. (1991) : Das niederrheinisch-westfälische Reichsgrafenkollegium und seine Mitglieder (1653-1806), Mayence.

Asbach, O. (2001a) : « Die Reichsverfassung als föderativer Staatenbund. Das Alte Reich in der politischen Philosophie des Abbé de Saint-Pierre und Jean-Jacques Rousseaus », in : Id. / Malettke, K. / Externbrink, S. (dir.) : Altes Reich, Frankreich und Europa. Politische, philosophische und historische Aspekte des französischen Deutschlandbildes im 17. und 18. Jahrhundert, Berlin, p. 171-218.

Asbach, O. (2001b) : « Zwischen Souveränität und Föderation : Moderne Staatlichkeit und die Ordnung Europas beim Abbé de Saint-Pierre und bei Jean-Jacques Rousseau ", Zeitschrift für Politikwissenschaft, 11, p. 1073-1099.

Asch, R. G. / Duchhardt, H. (dir.) (1996) : Der Absolutismus - ein Mythos? Strukturwandel monarchischer Herrschaft, Cologne / Weimar / Vienne.

Aulinger, R. (1980) : Das Bild des Reichstages im 16. Jahrhundert, Göttingen.

Bauer, J. (1997) : «Studentische Festerwartungen : Das Wartburgfest 1817 », in : Bünz, E. [et al.] (dir.) : Der Tag X in der Geschichte : Erwartungen und Enttäuschungen seit tausend Jahren, Stuttgart, p. $145-186$.

Behringer, W. (1990) : Thurn und Taxis. Die Geschichte ihrer Post und ihrer Unternehmen, Munich.

Behringer, W. (1998) : « Kommunikationswesen und territoriale Identität in der Frühen Neuzeit », in : Bellabarba, M. / Stauber, R. (dir.) : Territoriale Identität und politische Kultur in der Frühen Neuzeit, Berlin, p. 133-143.

Beik, W. (1985) : Absolutism and Society in Seventeenth-Century France : State Power and Provincial Aristocracy in Languedoc, New York.

Biedermann, K. (1883) : « Der Plan einer deutschen Zolleinheit im 16. Jahrhundert », Vierteljahrsschrift für Volkswirtschaft, Politik und Kulturgeschichte, 20, p. 41-56. 
Biefang, A. (1996) : « Der Streit um Treitschkes “Deutsche Geschichte” 1882/83 », Historische Zeitschrift, 262, p. 391-422.

Billinger Jr., R. D. (1998) : « Good and True Germans. The "Nationalism” of the Rheinbund Princes, 1806-1814 », in : Duchhardt, H. / Kunz, A. (dir.) : Reich oder Nation? Mitteleuropa 1780-1815, Mayence, p. 105-139.

Blickle, P. (1988) : Unruhen in der ständischen Gesellschaft 1300-1800, Munich.

Blickle, P. (2000) : Compte rendu de Georg Schmidt : Geschichte des Alten Reiches. Staat und Nation in der Frühen Neuzeit 1495-1806, Munich : C.H. Beck 1999, Neue Zürcher Zeitung, édition du $29 / 03$.

Böhme, E. (1989) : Das fränkische Reichsgrafenkollegium im 16. und 17. Jahrhundert. Untersuchungen zu den Möglichkeiten und Grenzen der korporativen Politik mindermächtiger Reichsstände, Stuttgart.

Bog, I. (1959) : Der Reichsmerkantilismus. Studien zur Wirtschaftspolitik des Heiligen Römischen Reiches im 17. und 18. Jahrhundert, Stuttgart.

Bogdandy, A. von (1991) : « Hegel und der Nationalstaat », Der Staat, 30, p. 513-525.

Bosbach, F. (dir.) (1992) : Feindbilder. Die Darstellung des Gegners in der politischen Publizistik des Mittelalters und der Neuzeit, Köln / Weimar / Vienne.

Brady Jr., T. (1997) : The Protestant Reformation in German History, German Historical Institute Washington, Annual Lecture.

Brechenmacher, T. (1996) : Großdeutsche Geschichtsschreibung im neunzehnten Jahrhundert. Die erste Generation (1830-48), Berlin.

Brockliss, L. [et al.] (dir.) (1997) : A Union of Multiple Identities : The British Isle c. 1750-c.1850, Manchester.

Buchheim, K. (1961) : « Das nationalstaatliche Denken im Deutschland des 19. und 20. Jahrhunderts ", in : Forster, K. (dir.) : Gibt es ein deutsches Geschichtsbild ?, Würzburg, p. $127-162$.

Burg, P. (1989) : Die deutsche Trias in Idee und Wirklichkeit. Vom Alten Reich zum deutschen Zollverein, Stuttgart.

Burgdorf, W. (1998) : Reichskonstitution und Nation. Verfassungsreformprojekte für das Heilige Römische Reich deutscher Nation im politischen Schriftum von 1648 bis 1806, Mayence.

Burgdorf, W. (1999) : “Chimäre Europa”. Antieuropäische Diskurse in Deutschland (1648-1999), Bochum. Burgdorf, W. (2000) : Compte rendu de Georg Schmidt : Geschichte des Alten Reiches. Staat und Nation in der Frühen Neuzeit 1495-1806, Munich : C.H. Beck 1999, Perform, 1 (3) (http:// www.sehepunkte.de/perform/review.php?id=38, consulté le 14 mars 2013).

Burgdorf, W. (2001a) : Compte rendu de Olav Moormann van Kappen / Dieter Wyduckel (dir.) : Der Westfälische Frieden in rechts- und staatstheoretischer Perspektive, Berlin 1998, Perform, 2 (5) (http://www.sehepunkte.de/perform/review.php?id=172, consulté le 25 mars 2013).

Burgdorf, W. (2001b) : « Die Geschichte der Versuche, die Verfassung des Heiligen Römischen Reiches Deutscher Nation zu reformieren ", in : Brendecke, A. / Burgdorf, W. (éd.) : Wege in die frühe Neuzeit, Neuried, p. 257-280.

Burkhardt, J. (en coll. avec J. Schumann) (1997) : « Reichskriege in der frühneuzeitlichen Bildpublizistik », in : Müller, R. A. (éd.) : Bilder des Reiches, Sigmaringen, p. 52-95. 
Burkhardt, J. (1999) : « Über das Recht der Frühen Neuzeit, politisch interessant zu sein », Geschichte in Wissenschaft und Unterricht,50, p. 748-756.

Buschmann, A. (dir.) (1984) : Kaiser und Reich, Munich.

Croxton, D. (1999) : « The Peace of Westphalia of 1648 and the Origins of Sovereignty ", The International History Review, 21, p. 569-591.

Dann, O. (2001) : « Geschichte Deutschlands », in : Maurer, M. (dir.) : Aufriß der Historischen Wissenschaften, vol. 2 : Räume, Stuttgart, p. 266-348.

Dehio, L. (1961) : « Der Zusammenhang der preußisch-deutschen Geschichte 1640-1945 », in : Forster, K. (dir.) : Gibt es ein deutsches Geschichtsbild ?, Würzburg, p. 67-90.

Dickmann, F. (1998) : Der Westfälische Frieden, 7e éd., Münster.

Diestelkamp, B. (dir.) (1990) : Das Reichskammergericht in der deutschen Geschichte, Cologne / Vienne.

Diestelkamp, B. (dir.) (1993) : Die politische Funktion des Reichskammergerichts, Cologne / Weimar / Vienne.

Dippel, H. (dir.) (1991) : Die Anfänge des Konstitutionalismus in Deutschland. Texte deutscher Verfassungsentwürfe am Ende des 18. Jahrhunderts, Francfort-sur-le-Main.

Dotzauer, W. (1998) : Die deutschen Reichskreise (1383-1806), Stuttgart.

Droysen, G. (1868) : Geschichte der preußischen Politik, partie 1, 2. éd., Leipzig (paru d'abord en 1855).

Duchhardt, H. (1990) : Altes Reich und europäische Staatenwelt 1648-1806, Munich.

Duchhardt, H. (1997) : Balance of Power und Pentarchie. Internationale Beziehungen 1700-1785, Paderborn.

Duggan, L. G. (1989) : «Zur Bedeutung des spätmittelalterlichen Kreditsystems für die frühneuzeitliche deutsche Geschichte », in : Schmidt, G. (dir.) : Stände und Gesellschaft im Alten Reich, Stuttgart, p. 201-209.

Duhamelle, C. (2000) : Compte rendu de Georg Schmidt : Geschichte des Alten Reiches. Staat und Nation in der Frühen Neuzeit 1495-1806, Munich : C.H. Beck 1999, Bulletin d'Information de la Mission Historique Française en Allemagne, 36, p. 285 sq.

Eberan, B. (1983) : Luther ? Friedrich der Große? Wagner ? Nietzsche? ... ? Wer war an Hitler schuld ? Die Debatte um die Schuldfrage 1945-1949, Munich.

Echternkamp, J. (1998) : Der Aufstieg des deutschen Nationalismus (1770-1840), Francfort-sur-leMain / New York.

Echternkamp, J. (2001) : “"Religiöses Nationalgefühl” oder “ Frömmelei der Deutschtümer” ? Religion, Nation und Politik im Frühnationalismus », in : Haupt, H.-G. / Langewiesche, D. (dir.) : Nation und Religion in der deutschen Geschichte, Francfort-sur-le-Main / New York, p. 142-169.

Edel, A. (1997) : Der Kaiser und Kurpfalz. Eine Studie zu den Grundelementen politischen Handelns bei Maximilian II. (1564-1576), Göttingen.

Elliott, J. A. (1992) : « A Europe of Composite Monarchies », Past \& Present, 127, p. 48-71.

Ellwein, T. (1992) : «Staatlichkeit im Wandel : das Staatsmodell des 19. Jahrhunderts als Verständnisbarriere », in : Kohler-Koch, B. (éd.) : Staat und Demokratie in Europa : 
18. Wissenschaftlicher Kongreß der Deutschen Vereinigung für Politische Wissenschaft, Leverkusen, p. 73-82.

Ernstberger, A. (1932) : Österreich-Preußen von Basel bis Campoformio 1795-1797, Prag.

Externbrink, S. (2001) : « Frankreich und die Reichsexekution gegen Friedrich II. Zur Wahrnehmung der Reichsverfassung durch die französische Diplomatie während des Siebenjährigen Krieges », in : Asbach, O. / Malettke, K. / Externbrink, S. (dir.) : Altes Reich, Frankreich und Europa. Politische, philosophische und historische Aspekte des französischen Deutschlandbildes im 17. und 18. Jahrhundert, Berlin, p. 221-253.

Faulenbach, B. (1980) : Ideologie des deutschen Weges. Die deutsche Geschichte in der Historiographie zwischen Kaiserreich und Nationalsozialismus, Munich.

Fichte, J. G. (1971 [1846]) : Fichtes Werke, éd. par. I. H. Fichte, rééd, vol. 7 : Zur Politik, Moral und Philosophie der Geschichte, Berlin.

Fichte, J. G. (1992) : Discours à la nation allemande, trad. A. Renault, Paris : Imprimerie Nationale.

Fischer, B. (2000) : «Fichte und der andere Weg in die Moderne », in : Vazsonyi, N. (dir.) : Searching for Common Ground. Diskurse zur deutschen Identität 1750-1871, Cologne / Weimar / Vienne, p. 61-75.

Friedeburg, R. von (2000) : «Welche Wegscheide in die Neuzeit ? Widerstandsrecht, "Gemeiner Mann" und konfessioneller Landespatriotismus zwischen "Münster" und "Magdeburg" ", Historische Zeitschrift, 270, p. 561-616.

Friedrich, K. (1999) : « Konfessionalisierung und politische Ideen in Polen-Litauen (1570 - 1650) », in : Bahlcke, J. / Strohmeyer, A. (dir.) : Konfessionalisierung in Ostmitteleuropa, Stuttgart, p. 249-265.

Gabel, H. (1995) : Widerstand und Kooperation. Studien zur politischen Kultur rheinischer und maasländischer Kleinterritorien (1648-1794), Tübingen.

Gaupp, F. (1948) : Die Fälschung der abendländischen Reichsidee, Baden-Baden.

Gerlach, I. / Nitschke, P. (dir.) (2000) : Metamorphosen des Leviathan? Staatsaufgaben im Umbruch, Opladen.

Gittel, U. (1996) : Die Aktivitäten des Niedersächsischen Reichskreises in den Sektoren »Friedenssicherung« und »Policey« (1554-1682), Hannovre.

Glagow, M. (dir.) (1984) : Gesellschaftssteuerung zwischen Korporatismus und Subsidiarität, Bielefeld.

Gräf, H. T. / Jendorff, A. / Pühringer, A. (2001) : « Staatsgewalt im Alten Reich der Neuen Zeit? Bemerkungen zu drei Neuerscheinungen und ihrer Bedeutung für die Landesgeschichte », Hessisches Jahrbuch für Landesgeschichte, 51, p. 257-267.

Graf, F. W. (2000) : « Die Nation - von Gott “erfunden” ? ", in : Krumeich, G. / Lehmann, H. (dir.) : "Gott mit uns". Nation, Religion und Gewalt im 19. und frühen 20. Jahrhundert, Göttingen, p. 285-317.

Grassmann, A. (2000) : “ "Die freie Übereinstimmung von Staat und Religion" - Hegel über Genese und Grund des modernen Staates ", in : Weisser-Lohmann, E. / Köhler, D. (dir.) : Verfassung und Revolution. Hegels Verfassungskonzeption und die Revolutionen der Neuzeit, Hambourg, p. 123-136.

Häpke, R. (1926) : « Der nationalwirtschaftliche Gedanke in Deutschland zur Reformationszeit », Historische Zeitschrift, 134, p. 350-368.

Härter, K. (1992) : Reichstag und Revolution 1789-1806, Göttingen.

Härter, K. (1993) : « Entwicklung und Funktion der Policeygesetzgebung des Heiligen Römischen Reiches Deutscher Nation im 16. Jahrhundert », Ius Commune, 20, p. 61-141. 
Hammerstein, N. (1972) : Jus und Historie, Göttingen.

Hammerstein, N. (1985) : « Zur Geschichte und Bedeutung der Universitäten im Heiligen Römischen Reich Deutscher Nation », Historische Zeitschrift, 241, p. 287-328.

Hammerstein, N. (dir.) (1995) : Staatslehre in der Frühen Neuzeit, Francfort-sur-le-Main.

Hansemann, D. (1919) : « Denkschrift über Preußens Lage und Politik (1830) », in : Hansen, J. (éd.) : Rheinische Briefe und Akten zur Geschichte der politischen Bewegung 1830-1850, vol. 1, Essen, p. 11-81.

Hanstein, W. (1947) : Von Luther bis Hitler. Ein wichtiger Abriß deutscher Geschichte, Dresden.

Hardtwig, W. (1994) : « Studentische Mentalität - Politische Jugendbewegung - Nationalismus. Die Anfänge der deutschen Burschenschaft », in : Id. : Nationalismus und Bürgerkultur in Deutschland 1500-1914. Ausgewählte Aufsätze, Göttingen, p. 108-148.

Hartmann, P. C. (1993) : «Bereits erprobt : Ein Mitteleuropa der Regionen », Das Parlament, édition du 03 au 10/12, p. 21.

Hartmann, P. C. (1997) : Der Bayerische Reichskreis (1500 bis 1803), Berlin.

Hattenhauer, H. (1990) : Geschichte der deutschen Nationalsymbole, 2e éd., Munich.

Heckel, M. (1983) : Deutschland im konfessionellen Zeitalter, Göttingen.

Heeren, A. H. L. (1819) : Handbuch der Geschichte des Europäischen Staatensystems und seiner Colonien, 3e éd., Göttingen (première parution en 1809).

Hegel, G. F. Wilhelm (1998) : « Fragmente seiner Kritik der Verfassung Deutschlands

(1799-1803) », in : Id. : Gesammelte Werke, éd. par M. Baum u. K. Meist, Hambourg, vol. 5, p. 1-202.

Heil, D. (1998) : Die Reichspolitik Bayerns unter der Regierung Herzog Albrechts V. (1550-1579), Göttingen.

Heller, H. (1921) : Hegel und der nationale Machtstaatsgedanke in Deutschland, Leipzig, rééd. Aalen 1963.

Hess, W. (1997) : « Reichsikonographie auf Münzen der Neuzeit », in : Müller, R. A. (éd.) : Bilder des Reiches, Sigmaringen, p. 169-187.

Hintze, O. (1970) : «Wesen und Wandlung des modernen Staats ", in : Id. : Staat und Verfassung. Gesammelte Abhandlungen zur allgemeinen Verfassungsgeschichte, dir. par G. Oestreich, 3e éd., Göttingen, p. 470-496.

Hollenbeck, M. (1999) : « Die Türkenpublizistik im 17. Jahrhundert - Spiegel der Verhältnisse im Reich ?», Mitteilungen des Instituts für österreichische Geschichtsforschung, 107, p. 111-130.

Hoover, A. J. (1986) : The Gospel of Nationalism: German Patriotic Preaching from Napoleon to Versailles, Stuttgart.

Huhn, J. (1990) : Lernen aus der Geschichte? Historische Argumente in der westdeutschen Föderalismusdiskussion 1945-1949, Melsungen.

Hye, F.-H. (1973) : « Der Doppeladler als Symbol für Kaiser und Reich », Mitteilungen des Instituts für österreichische Geschichtsforschung, 81, p. 63-100.

Immich, M. (1905) : Geschichte des europäischen Staatensystems von 1660 bis 1789, Munich / Berlin.

Jeismann, M. (1992) : Das Vaterland der Feinde: Studien zum nationalen Feindbegriff und Selbstverständnis in Deutschland und Frankreich, Stuttgart. 
Jörn, N. / North, M. (dir.) (2000) : Die Integration des südlichen Ostseeraums in das Alte Reich, Cologne / Weimar / Vienne.

Johnston, O. W. (1990) : Der deutsche Nationalmythos. Ursprung eines politischen Programms, Stuttgart. Kaiser, G. (1973) : Pietismus und Patriotismus im literarischen Deutschland, Francfort-sur-le-Main. Kemiläinen, A. (1956) : Auffassungen über die Sendung des deutschen Volkes um die Wende des 18. und 19. Jahrhunderts, Helsinki.

Kiessling, R. (1997) : « Under des Römischen Adlers Flügel ... Das schwäbische Judentum und das Reich », in : Müller, R. A. (éd.) : Bilder des Reiches, Sigmaringen, p. 221-253.

Klinger, A. (2002) : Der Gothaer Fürstenstaat, Husum.

Koenigsberger, H. G. (1991) : « Zusammengesetzte Staaten, Repräsentativversammlungen und der amerikanische Unabhängigkeitskrieg », Zeitschrift für Historische Forschung, 18, p. 399-423.

Kohler, A. (1990) : Das Reich im Kampf um die Hegemonie in Europa 1521-1648, Munich.

Koselleck, R. (1999) : « Deutschland - eine verspätete Nation ? », in : Id. : Europäische Umrisse deutscher Geschichte, Heidelberg, p. 37-78.

Krieger, L. (1957) : The German Idea of Freedom, Boston.

Kriele, M. (1975) : Einführung in die Staatslehre, Reinbek.

Krüger, K. (1980) : Finanzstaat Hessen 1500-1567. Staatsbildung im Übergang vom Domänenstaat zum Steuerstaat, Marburg.

Lange, H. (2000) : « The Nation-State and the Holy Roman Empire : Goethe and Moser Reconsidered », in : Vazsonyi, N. (dir.) : Searching for Common Ground. Diskurse zur deutschen Identität 1750-1871, Cologne /Weimar / Vienne, p. 141-159.

Langewiesche, D. (2000a) : « Föderativer Nationalismus als Erbe der deutschen Reichsnation. Über Föderalismus und Zentralismus in der deutschen Nationalgeschichte », in : Id. / Schmidt, G. (dir.) : Föderative Nation. Deutschlandkonzepte von der Reformation bis zum Ersten Weltkrieg, Munich, p. 215-242.

Langewiesche, D. (2000b) : Nation, Nationalismus, Nationalstaat in Deutschland und Europa, Munich.

Lanzinner, M. (1993) : Friedenssicherung und politische Einheit des Reiches unter Kaiser Maximilian II. (1564-1576), Göttingen.

Lindgren, U. (1997) : « Die Grenzen des Alten Reiches auf gedruckten Karten », in : Müller, R. A. (dir.) : Bilder des Reiches, Sigmaringen, p. 31-50.

Luttenberger, A. P. (1994) : Kurfürsten, Kaiser und Reich. Politische Führung und Friedenssicherung unter Ferdinand I. und Maximilian II., Mayence.

Lutz, H. (1958) : Conrad Peutinger. Beiträge zu einer politischen Biographie, Augsburg.

Lutz, H. (1964) : Christianitas afflicta. Europa, das Reich und die päpstliche Politik im Niedergang der Hegemonie Kaiser Karls V. (1552-1556), Göttingen.

Mader, E.-O. (2001) : « Das Alte Reich in neuem Licht. Perspektiven auf sein Ende und sein Nachwirken im frühen 19. Jahrhundert », in : Brendecke, A. / Burgdorf, W. (dir.) : Wege in die Frühe Neuzeit, Neuried, p. 235-256.

Maier, H. (1992) : Das Freiheitsproblem in der deutschen Geschichte, Heidelberg.

Meinecke, F. (1908) : Weltbürgertum und Nationalstaat, Munich (paru d'abord en 1907). 
Meissner, E. (1949) : Zwiespalt im Abendland. Ein Kommentar zur deutschen Geschichte von 1517-1939, Stuttgart.

Möser, J. (1842) : « Vorschlag zu einem neuen Plan der deutschen Reichsgeschichte », in : Id. : Patriotische Phantasien, vol. 4, Berlin.

Moraw, P. (1988) : « Über Patrone und Klienten im Heiligen Römischen Reich des späten Mittelalters und der frühen Neuzeit », in : Maczak, A. (dir.) : Klientelsysteme im Europa der Frühen Neuzeit, Munich, p. 1-18.

Moser, J. J. (1766) : Neues Teutsches Staatsrecht, vol. 1, Stuttgart.

Müller, H. (1989) : « Deutscher Bund und deutsche Nationalbewegung », Historische Zeitschrift, 248, p. 51-78.

Müller, K. (1973) : « Zur Reichskriegserklärung im 17. und 18. Jahrhundert », Zeitschrift der Savigny-Stiftung für Rechtsgeschichte, Germ. Abt., 90, p. 246-259.

Müller, R. A. (1997) : « Das “ Heilige Römische Reich Deutscher Nation” in allegorischen Darstellungen », in : Id. (éd.) : Bilder des Reiches, Sigmaringen, p. 397-432.

Münkler, H. / Grünberger, H. (1998) : « Arminius/Hermann als nationales Symbol im Diskurs der deutschen Humanisten (1500-1570) », in : Münkler, H. / Grünberger, H. / Mayer, K. (dir.) : Nationenbildung : die Nationalisierung Europas im Diskurs humanistischer Intellektueller, Italien und Deutschland, Berlin, p. 263-308.

Muhlack, U. (1989) : « Die Germania im deutschen Nationalbewußtsein vor dem 19. Jahrhundert », in : Jankuhn, H. / Timpe, D. (dir.) : Beiträge zum Verständnis des Tacitus, vol. 1, Göttingen, p. 128-154.

Neugebauer, W. (2001) : Zentralprovinz im Absolutismus, Brandenburg im 17. und 18. Jahrhundert, Berlin.

Neugebauer-Wölk, M. (1998) : « Reich oder Republik ? Pläne und Ansätze zur Republikanischen Neugestaltung im Alten Reich 1790-1800», in : Duchhardt, H. / Kunz, A. (dir.) : Reich oder Nation? Mitteleuropa 1780-1815, Mayence, p. 21-50.

Neuhaus, H. (1982) : Reichsständische Repräsentationsformen im 16. Jahrhundert. Reichstag Reichskreistag - Reichsdeputationstag, Berlin.

Neuhaus, H. (1986) : « Das Problem der militärischen Exekutive in der Spätphase des Alten Reiches », in : Kunisch, J. (dir.) : Staatsverfassung und Heeresverfassung in der europäischen Geschichte der frühen Neuzeit, Berlin, p. 297-346.

Neuhaus, H. (1989) : « Das Reich im Kampf gegen Friedrich den Großen », in : Kroener, B. R. (dir.) : Europa im Zeitalter Friedrichs des Großen, Munich, p. 213-243.

Neuhaus, H. (1997) : Das Reich in der Frühen Neuzeit, Munich.

Nipperdey, T. (1983) : Deutsche Geschichte 1800-1866, vol. 1 : Bürgerwelt und starker Staat, Munich.

Oestmann, P. (2000) : Compte rendu de Georg Schmidt : Geschichte des Alten Reiches. Staat und Nation in der Frühen Neuzeit 1495-1806, Munich : C.H. Beck 1999, Ius Commune, 27, p. 483-486.

Oestreich, G. (1969) : Geist und Gestalt des frühmodernen Staates, Berlin.

Oestreich, G. (1980) : Strukturprobleme der Frühen Neuzeit, Berlin.

Ogilvie, S. (1999) : «The State in Germany. A Non-Prussian View », in : Brewer, J. / Hellmuth, E. : Rethinking Leviathan. The Eighteenth-Century State in Britain and Germany, Oxford, p. 167-202. 
Ott, T. (2001) : « Das Alte Reich im späten 16. Jahrhundert. Das Problem seiner "Staatlichkeit" am Beispiel der Beziehungen zu Ostmitteleuropa ", in : Brendecke, A. / Burgdorf, W. (dir.) : Wege in die Frühe Neuzeit, Neuried, p. 187-214.

Perceval-Maxwell, M. (1991) : «Ireland and the Monarchy in the Early Stuart Multiple Kingdom », The Historical Journal, 34, p. 279-295.

Plassmann, M. (2000) : Krieg und Defension am Oberrhein. Die Vorderen Reichskreise und Markgraf Ludwig Wilhelm von Baden (1693-1706), Berlin.

Plessner, H. (1959) : Die verspätete Nation. Über die Verführbarkeit bürgerlichen Geistes, Stuttgart.

Pütter, J. S. (1777) : Beyträge zum Teutschen Staats- und Fürsten-Rechte, vol. 1, Göttingen.

Press, V. (1970) : Calvinismus und Territorialstaat. Regierung und Zentralbehörden der Kurpfalz 1559-1619, Stuttgart.

Press, V. (1986) : « The Habsburg Court as Center of the Imperial Government », Journal of Modern History, 58 (Supplementum : Politics and Society in the Holy Roman Empire 1500-1806), p. 523-545.

Press, V. (1987) : Das Reichskammergericht in der deutschen Geschichte, Wetzlar.

Press, V. (1988a) : « Patronat und Klientel im Heiligen Römischen Reich », in : Maczak, A. (dir.) : Klientelsysteme im Europa der Frühen Neuzeit, Munich, p. 19-46.

Press, V. (1988b) : « Der Untergang des Heiligen Römischen Reiches deutscher Nation », in : Müller, E. (dir.) : »... aus der anmuthigen Gelehrsamkeit«. Dietrich Geyer zum 60. Geburtstag, Tübingen, p. 81-97.

Press, V. (1989) : « Die kaiserliche Stellung im Reich zwischen 1648 und 1740 - Versuch einer Neubewertung ", in : Schmidt, G. (dir.) : Stände und Gesellschaft im Alten Reich, Stuttgart, p. 51-80.

Press, V. (1991) : «The Imperial Court of the Habsburgs. From Maximilian I to Ferdinand III, 1493-1657 », in : Asch, R. G. / Birke, A. M. (dir.) : Princes, Patronage and the Nobility, Oxford, p. 289-312.

Press, V. (1997) : « Das Römisch-deutsche Reich - ein politisches System in verfassungs- und sozialgeschichtlicher Fragestellung », in : Id. : Das Alte Reich. Ausgewählte Aufsätze, éd. par J. Kunisch, Berlin, p. 18-41.

Press, V. (1998a) : « Reichsgrafenstand und Reich », in : Id. : Adel im Alten Reich, Tübingen, p. 113-138.

Press, V. (1998b) : « Die Reichsritterschaft im Reich der Frühen Neuzeit », in : Id. : Adel im Alten Reich, Tübingen, p. 205-231.

Raumer, K. von (1952) : « Saint Pierre und Rousseau. Das Problem des Ewigen Friedens », Zeitschrift für die gesamte Staatswissenschaft,108, p. 669-689.

Rauscher, P. / Staudinger, B. (2000) : « Der Staat in der Frühen Neuzeit. Überlegungen und Fragen zu aktuellen Neuerscheinungen der deutschen Geschichtswissenschaft », Mitteilungen des Österreichischen Staatsarchivs, 48, p. 405-424.

Reden-Dohna, A. von (1982) : Reichsstandschaft und Klosterherrschaft. Die schwäbischen Reichsprälaten im Zeitalter des Barock, Wiesbaden.

Reinhard, W. (1999) : Geschichte der Staatsgewalt, Munich. 
Reinhard, W. (2001) : « Geschichte als Delegitimation », Frankfurter Allgemeine Zeitung, édition du $16 / 11$, p. 45.

Roeck, B. (1984) : Reichssystem und Reichsherkommen. Die Diskussion über die Staatlichkeit des Reiches in der politischen Publizistik des 17. und 18. Jahrhunderts, Stuttgart.

Rokkan, S. (1966) : « Norway : Numerical Democracy and Corporate Pluralism », in : Dahl, R. A. (dir.) : Political Opposition in Western Democracies, New Haven, p. 70-115.

Sailer, R. (1999) : Untertanenprozesse vor dem Reichskammergericht. Rechtsschutz gegen die Obrigkeit in der zweiten Hälfte des 18. Jahrhunderts, Cologne / Weimar / Vienne.

Scheurmann, I. (dir.) (1994) : Frieden durch Recht. Das Reichskammergericht von 1495 bis 1806, Ausstellungskatalog, Mayence.

Schiller, F. (1983) : [Deutsche Größe], in : Schillers Werke, Nationalausgabe 2.1, Weimar, p. 431-436. Schilling, H. (1988) : « Gab es im späten Mittelalter und zu Beginn der Neuzeit in Deutschland einen städtischen " Republikanismus" ? Zur politischen Kultur des alteuropäischen Stadtbürgertums », in : Koenigsberger, H. G. (dir.) : Republiken und Republikanismus im Europa der Frühen Neuzeit, Munich, p. 101-143.

Schilling, H. (1991) : « Stadt und frühmoderner Territorialstaat : Stadtrepublikanismus versus Fürstensouveränität », in : Stolleis, M. (dir.) : Recht, Verfassung und Verwaltung in der frühneuzeitlichen Stadt, Cologne / Vienne, p. 19-39.

Schilling, H. (1999) : Die neue Zeit. Vom Christeneuropa zum Europa der Staaten, 1250 bis 1750, Berlin. Schilling, H. (2000) : «Wider den Mythos vom Sonderweg - die Bedingungen des deutschen Weges in die Neuzeit", in : Heinig, P.-J. [et al.] (dir.) : Reich, Regionen und Europa in Mittelalter und Neuzeit. Festschrift für Peter Moraw, Berlin, p. 699-714.

Schilling, H. (2001) : « Reichs-Staat und frühneuzeitliche Nation der Deutschen oder teilmodernisiertes Reichssystem. Überlegungen zu Charakter und Aktualität des Alten Reiches ", Historische Zeitschrift, 272, p. 377-395.

Schilling, M. (1990) : Bildpublizistik der frühen Neuzeit, Tübingen.

Schindling, A. / Ziegler, W. (dir.) (1990) : Die Kaiser der Neuzeit 1519-1918, Munich.

Schindling, A. (1991) : Die Anfänge des Immerwährenden Reichstags zu Regensburg. Ständevertretung und Staatskunst nach dem Westfälischen Frieden, Mayence.

Schindling, A. (2001) : « Kaiser, Reich und Reichsverfassung 1648-1806. Das neue Bild vom Alten Reich ", in : Asbach, O. / Malettke, K. / Externbrink, S. (dir.) : Altes Reich, Frankreich und Europa. Politische, philosophische und historische Aspekte des französischen Deutschlandbildes im 17. und 18. Jahrhundert, Berlin, p. 25-54.

Schlie, U. (1961) : Johann Stephan Pütters Reichsbegriff, Göttingen.

Schmale, W. (1998) : " "Grenze" in der deutschen und französischen Frühneuzeit », in : Schmale, W. / Stauber, R. (dir.) : Menschen und Grenzen in der Frühen Neuzeit, Berlin, p. 50-75.

Schmid, A. (2000) : Compte rendu de Georg Schmidt : Geschichte des Alten Reiches. Staat und Nation in der Frühen Neuzeit 1495-1806, Munich : C.H. Beck 1999, Zeitschrift für bayerische Landesgeschichte, 63, p. 991-993.

Schmid, P. (1983) : « Reichssteuer, Reichsfinanzen und Reichsgewalt in der ersten Hälfte des 16. Jahrhunderts », in : Angermeier, H. (dir.) : Säkulare Aspekte der Reformationszeit, Munich / Vienne, p. 153-199. 
Schmidt, A. (2007) : Vaterlandsliebe und Religionskonflikt. Politische Diskurse im Alten Reich (1555-1648), Leiden / Boston / Tokyo.

Schmidt, G. (1984) : Der Städtetag in der Reichsverfassung. Eine Untersuchung zur korporativen Politik der Freien und Reichsstädte in der ersten Hälfte des 16. Jahrhunderts, Stuttgart.

Schmidt, G. (1989) : Der Wetterauer Grafenverein. Organisation und Politik einer Reichskorporation zwischen Reformation und Westfälischem Frieden, Marburg.

Schmidt, G. (1991) : « Die frühneuzeitlichen Hungerrevolten », Zeitschrift für Historische Forschung, 18 , p. 257-280.

Schmidt, G. (1993) : « Der Rheinbund und die deutsche Nationalbewegung », in : Timmermann, H. (dir.) : Die Entstehung der Nationalbewegung in Europa 1750-1849, Berlin, p. 29-44.

Schmidt, G. (1995) : « Der napoleonische Rheinbund - ein erneuertes Altes Reich ? », in : Press, V. (dir.) : Alternativen zur Reichsverfassung in der Frühen Neuzeit ?, Munich, p. 227-246.

Schmidt, G. (1996) : « Deutschland am Beginn der Neuzeit. Reichs-Staat und Kulturnation », in : Roll, C. [et al.] (dir.) : Recht und Reich im Zeitalter der Reformation. Festschrift für Horst Rabe, Francfort-sur-le-Main, p. 1-30.

Schmidt, G. (1997) : «"Wo Freiheit ist und Recht ...”, da ist der Deutsche Untertan ? », in : Werner, M. (dir.) : Identität und Geschichte, Weimar, p. 105-124.

Schmidt, G. (1998) : « Der Westfälische Friede als Grundgesetz des komplementären ReichsStaats ", in : Bussmann, K. / Schilling, H. (dir.) : 1648 - Krieg und Frieden in Europa, catalogue d'exposition, vol. de textes 1 : Politik, Religion, Recht und Gesellschaft, Münster, p. 447-454.

Schmidt, G. (1999) : Geschichte des Alten Reiches. Staat und Nation in der Frühen Neuzeit 1495-1806, Munich.

Schmidt, G. (2000) : « Teutsche Kriege : Nationale Deutungsmuster und integrative Wertvorstellungen im frühneuzeitlichen Reich», in : Langewiesche, D. / Schmidt, G. (dir.) : Föderative Nation. Deutschlandkonzepte von der Reformation bis zum Ersten Weltkrieg, Munich, p. 33-61.

Schmidt, G. (2001a) : « "Absolutes Dominat” oder "deutsche Freiheit”. Der Kampf um die Reichsverfassung zwischen Prager und Westfälischem Frieden », in : Friedburg, R. von (dir.) : Widerstandsrecht in der frühen Neuzeit, Berlin, p. 265-284.

Schmidt, G. (2001b) : « Die "deutsche Freiheit” und der Westfälische Friede », in : Asch, R. G. [et al.] (dir.) : Frieden und Krieg in der Frühen Neuzeit, Munich, p. 323-347.

Schmidt, G. (2001c) : « Das frühneuzeitliche Reich - komplementärer Staat und föderative Nation », Historische Zeitschrift, 273, p. 371-400.

Schmidt, G. (2002) : « Friedrich Schillers "Deutsche Größe" und der nationale Universalismus », in : Greiling, W. / Hahn, H.-W. (dir.) : Tradition und Umbruch. Geschichte zwischen Wissenschaft, Kultur und Politik, Rudolstadt, p. 11-32.

Schmidt, H. (1988) : « Onno Klopp und die "kleindeutschen Geschichtsbaumeister” », in : Portmann-Tinguely, A. (dir.) : Kirche, Staat und katholische Wissenschaft in der Neuzeit, Paderborn, p. 381-395.

Schmidt, H. R. (1986) : Reichsstädte, Reich und Reformation. Korporative Religionspolitik 1521-1529/30, Stuttgart.

Schmidt, H. R. (1997) : « Sozialdisziplinierung ? », Historische Zeitschrift, 265, p. 639-682. 
Schmidt, P. (2001) : Spanische Universalmonarchie oder »teutsche Libertet«. Das spanische Imperium in der Propaganda des Dreißigjährigen Krieges, Stuttgart.

Schnettger, M. (2002) : « Impero romano - Impero germanico. Italienische Perspektiven auf das Reich in der Frühen Neuzeit », in : Id. (dir.) : Imperium Romanum - irregulare corpus - Teutscher Reichs-Staat? Das Alte Reich im Verständnis der Zeitgenossen und der Historiographie, Mayence, p. 53-75.

Schönemann, B. (1989) : « Nationale Identität als Aufgabe des Geschichtsunterrichts nach der Reichsgründung », Internationale Schulbuchforschung, 11, p. 107-128.

Schorn-Schütte, L. (1993) : «E. Troeltschs Soziallehre und die gegenwärtige Frühneuzeitforschung. Zur Diskussion um die Bedeutung von Luthertum und Calvinismus für die Entstehung der modernen Welt », in : Graf, F. W. / Rendtorff, T. (dir.) : Ernst Troeltschs Soziallehren, Gütersloh, p. 133-151.

Schräder, W. H. (1996) : «Zum Verhältnis von Staat und Nation im Spätwerk J. G. Fichtes », in : Burger, R. / Klein, H.-D. / Schräder, W. H. (dir.) : Gesellschaft, Staat, Nation, Vienne, p. 7-18.

Schrader, F. E. (2001) : «Politisch-semantische Strategien vom Ancien Regime zur Restauration. Das Alte Reich als (Con)fédération, Corps, Système in der französischen Publizistik ", in : Asbach, O. / Malettke, K. / Externbrink, S. (dir.) : Altes Reich, Frankreich und Europa. Politische, philosophische und historische Aspekte des französischen Deutschlandbildes im 17. und 18. Jahrhundert, Berlin, p. 105-119.

Schubert, F. H. (1966) : Die deutschen Reichstage in der Staatslehre der frühen Neuzeit, Göttingen.

Schuck, G. (1994) : Rheinbundpatriotismus und politische Öffentlichkeit zwischen Aufklärung und Frühliberalismus, Stuttgart.

Schulze, W. (1975) : « Die veränderte Bedeutung sozialer Konflikte im 16. und 17. Jahrhundert », in : Wehler, H.-U. (dir.) : Der deutsche Bauernkrieg 1524-26, Göttingen, p. 277-302.

Schulze, W. (1978) : Reich und Türkengefahr im späten 16. Jahrhundert, Munich.

Schulze, W. (1980) : Bäuerlicher Widerstand und feudale Herrschaft in der frühen Neuzeit, Stuttgart-Bad Cannstatt.

Siemann, W. (1995) : Vom Staatenbund zum Nationalstaat. Deutschland 1806-1871, Munich.

Smend, R. (1911) : Das Reichskammergericht, Geschichte und Verfassung, Weimar.

Straub, E. (2001) : Eine kleine Geschichte Preußens, Berlin.

Stauber, R. (1998) : « "Auf der Grenzscheide des Südens und Nordens”. Zur Ideengeschichte der Grenze zwischen Deutschland und Italien », in : Schmale, W. / Stauber, R. (dir.) : Menschen und Grenzen in der Frühen Neuzeit, Berlin, p. 76-115.

Steiger, G. (1991) : Urburschenschaft und Wartburgfest. Aufbruch nach Deutschland, 2. éd., Leipzig.

Stein, K. Freiherr vom (1961) : Briefe und amtliche Schriften, vol. 3 : A Brünn (Brno) et Prague. La crise de 1811. A Moscou et Saint-Pétersbourg. Le grand tournant (1809-1812), éd. par W. Hubatsch, Stuttgart.

Stenzel, G. A. H. (1837) : Geschichte des preussischen Staats, 2e partie, Hambourg.

Treitschke, H. von (1882) : Deutsche Geschichte, vol. 1, 3. éd., Leipzig (paru d'abord en 1879).

Trossbach, W. (1985) : Bauernbewegungen im Wetterau-Vogelsberg-Gebiet 1648-1806, Darmstadt. 
Van Gelderen, M. (1996a) : « Holland und das Preußentum : Justus Lipsius zwischen niederländischem Aufstand und brandenburg-preußischem Absolutismus », Zeitschrift für Historische Forschung, 23, p. 29-56.

Van Gelderen, M. (1996b) : « Liberty, Civic Rights, and Duties in the Sixteenth-Century Europe and the Rise of the Dutch Republic ", in : Coleman, J. (dir.) : The Individual in Political Theory and Practice, Oxford, p. 99-122.

Vann, J. A. (1975) : The Swabian Kreis. Institutional Growth in the Holy Roman Empire, 1648-1715, Bruxelles.

Vann, J. A. (1986) : Württemberg auf dem Weg zum modernen Staat, 1593-1793, Stuttgart.

Vogt, R. (1995a) : « Der kooperative Staat. Krisenbewältigung durch Verhandlung ? », in : Id. (dir.) : Der kooperative Staat, Baden-Baden, p. 11-29.

Vogt, R. (1995b) : « Der kooperative Staat. Auf der Suche nach einem neuen Steuerungsmodus », in : Id. (dir.) : Der kooperative Staat, Baden-Baden, p. 33-92.

Volckart, O. (1999) : « Politische Zersplitterung und Wirtschaftswachstum im Alten Reich, ca. 1650-1800 », Vierteljahrsschrift für Sozial- und Wirtschaftsgeschichte, 86, p. 1-38.

Wagner, M. (1996) : « Germania und ihre Freier. Zur Herausbildung einer deutschen nationalen Ikonographie um 1800 », in : Herrmann, U. (dir.) : Volk - Nation - Vaterland, Hambourg, p. 244-268.

Weber, W. (1998) : « Dynastiesicherung und Staatsbildung. Die Entfaltung des frühmodernen Fürstenstaates », in : Id. (dir.) : Der Fürst. Ideen und Wirklichkeit in der europäischen Geschichte, Cologne / Weimar / Vienne, p. 91-136.

Wehler, H.-U. (1987) : Deutsche Gesellschaftsgeschichte, vol. 1, Munich.

Wehler, H.-U. (2001) : Nationalismus, Munich.

Weisert, H. (1994) : « Der Reichstitel bis 1806 », Archiv für Diplomatik, 40, p. 441-513.

Weisser-Lohmann, E. (2000) : «"Daß das Allgemeine zu einer Tat komme” - "Sittlichkeit” und "Verfassung” bei Hegel », in : Id. / Köhler, D. (dir.) : Verfassung und Revolution. Hegels Verfassungskonzeption und die Revolutionen der Neuzeit, Hambourg, p. 137-166.

Westphal, S. (2002) : Stabilisierung durch Recht. Reichsgerichtsbarkeit und thüringische Territorialstaaten (1648-1806), Cologne / Weimar / Vienne.

Whaley, J. (1996) : « Thinking about Germany, 1750-1815 : The Birth of a Nation ? », Publications of the English Goethe Society N. S., 66, p. 54-72.

Whaley, J. (2002) : « Federal Habits : The Holy Roman Empire and the Continuity of German Federalism », in : Umbach, M. (dir.) : German Federalism. Past, Present and Future, Houndmills [et al.], p. 15-41.

Wiarda, H. J. (1997) : Corporatism and Comparative Politics. The Other Great »Ism«, Armonk.

Wiegels, R. / Woesler, W. (dir.) (1995) : Arminius und die Varusschlacht. Geschichte, Mythos, Literatur, Paderborn.

Willoweit, D. (1984) : «Von der alten deutschen Freiheit », in : Heyn, E. V. (dir.) : Vom normativen Wandel des Politischen, Berlin, p. 17-42.

Winkler, H. A. (2000) : Der lange Weg nach Westen, 2 vol., Munich : C. H. Beck. 
Wolgast, E. (2000) : Compte rendu de Georg Schmidt : Geschichte des Alten Reiches. Staat und Nation in der Frühen Neuzeit 1495-1806, Munich : C.H. Beck 1999, Zeitschrift des Vereins für Thüringische Geschichte, 54, p. 341 sq.

Wolgast, E. (2002) : « Die Sicht des Alten Reiches bei Treitschke und Erdmannsdörffer », in : Schnettger, M. (dir.) : Imperium Romanum - irregulare corpus - Teutscher Reichs-Staat ? Das Alte Reich im Verständnis der Zeitgenossen und der Historiographie, Mayence, p. 169-188.

Wüst, W. (dir.) (2000) : Reichskreis und Territorium : Die Herrschaft über der Herrschaft ? Supraterritoriale Tendenzen in Politik, Kultur, Wirtschaft und Gesellschaft. Ein Vergleich süddeutscher Reichskreise, Stuttgart.

Zimmer, H. (1971) : Auf dem Altar des Vaterlandes. Religion und Patriotismus in der deutschen Kriegslyrik des 19. Jahrhunderts, Francfort-sur-le-Main.

Zorn, W. (1959) : « Reichs- und Freiheitsgedanken in der Publizistik des ausgehenden 18. Jahrhunderts (1763-1792) », in : Wentzcke, P. (dir.) : Darstellungen und Quellen zur Geschichte der deutschen Einheitsbewegung im neunzehnten und zwanzigsten Jahrhundert, Heidelberg, p. 11-66.

Zwiedineck-Südenhorst, H. von (1888) : Die öffentliche Meinung in Deutschland im Zeitalter Ludwigs XIV. 1650-1700, Stuttgart.

\section{NOTES}

1. Schmidt, G. (1999).

*. NDT : la traduction mot-à-mot de ce concept forgé par l'auteur de l'article entend conserver, dans sa relative lourdeur, l'adjectif «complémentaire» (voir plus loin dans la contribution l'explication qui en est donnée) renvoyant à une dynamique politique et territoriale animée par le couple État et Empire que relie le trait d'union. Une autre traduction aurait pu adopter l'expression d'«Empire comme État subsidiaire» que l'on trouve également dans l'historiographie française du Saint-Empire moderne.

2. Schilling, H. (2001). Voir aussi Schmidt, G. (2001c), plus spécialement les p. 378 sq. ; Ott (2001) ; Reinhard (2001). A compléter par Rauscher / Staudinger (2000); pour des postulats comparables sur la non-étaticité de l'Empire, voir : Gräf / Jendorff / Pühringer (2001).

3. Voir par exemple Oestmann (2000) ; Burgdorf (2000) ; Blickle (2000); Wolgast (2000) ; Schmid, A. (2000); Duhamelle (2000); Whaley (2002). Je remercie l'auteur pour m'avoir permis de consulter son texte avant l'édition.

*. NDT: on a traduit tout au long de l'article «frühneuzeitlich» et «Frühe Neuzeit» par " moderne » ou "époque moderne », conformément aux césures chronologiques académiques françaises qui placent les Temps "modernes" des XVIe-XVIIIe siècles entre le Moyen Âge et l'époque contemporaine. Le terme allemand "vormodern », d'une acception chronologique plus large, a été rendu par « prémoderne » et s'entend en rapport à la «Moderne » allemande restitué ici par la notion de « modernité ».

4. Sur la thèse du Sonderweg: Plessner (1959). Les ouvrages classiques relevant de cette historiographie sont Hanstein (1947) ; Meissner (1949) ; sur ces derniers : Eberan (1983) ; Brady Jr. (1997), en particulier la page 28. Rejetant au contraire la thèse de la voie particulière : Koselleck (1999), p. 37-78. Sur les fondements de cette interprétation: Faulenbach (1980). Récemment, Heinrich August Winkler a souligné la thèse d'une séparation de la «voie» occidentale en ouvrant sa magistrale synthèse par la célèbre phrase «Au commencement était l'Empire »: Winkler (2000), ici vol. 1, p. 5. 
*. NDT : on a choisi de traduire Sonderweg, dont l'usage s'est désormais francisé, par l'expression traditionnellement reçue de „voie particulière“ préférable à celle, plus tranchée et donc moins connectée à l'histoire européenne, de " chemin séparé ».

5. Schilling, H. (2001), p. 383. Et Schilling, H. (2000) ; Hartmann (1993).

*. NDT : En allemand le terme „altes Reich“ désigne le Saint-Empire dans son existence jusqu'en 1806. Dans cet article d'un moderniste, il sera presque toujours question de cet Empire pendant la période moderne, raison pour laquelle on a ensuite traduit "altes Reich» par Saint-Empire moderne.

6. Schilling, H. (2001), p. 394. On retrouve les mêmes expressions dans Schilling, H. (1999), p. 141. NDLR : voir l'article de H. Schilling traduit dans ce numéro de Trivium

7. Reinhard (1999), p. 55.

8. Voir Kriele (1975), p. 74 sq.

9. Rokkan (1966) ; Glagow (1984); Vogt (1995a), (1995b) ; Wiarda (1997) ; Gerlach / Nitschke (2000).

10. Voir sur ce point Asbach (2001b).

11. Ellwein (1992).

12. Vogt (1995a), p. 12

13. Hintze (1970), p. 476.

14. Van Gelderen (1996a), p. 45. - Cet article important relève que Juste Lipse ne peut pas être tenu, au sens où l'entend Gerhard Oestreich, pour un théoricien de l'État absolutiste. Voir aussi Asch / Duchhardt (1996).

15. Friedrich (1999), p. 251.

16. Burgdorf (1998). Voir aussi, dans une autre perspective Lange (2000).

17. Dippel (1991) ; Neugebauer-Wölk (1998).

18. Burgdorf (2001b). Sa conclusion ne peut être plus limpide : «Partant de la structuration même de l'Empire, à la question de savoir si celui-ci était bien un État à la fin du XVIIe et au XVIIIe siècle, la réponse est incontestablement oui » (ibid, p. 263).

*. En latin dans le texte [NDT].

19. Roeck (1984), p. 24 sq. et 45 sq.

20. Moser (1766), p. 550.

21. Pütter (1777), p. 30 sq. Voir aussi Schlie (1961).

22. Perceval-Maxwell (1991); Koenigsberger (1991); Elliott (1992); Brockliss (1997).

23. Voir aussi ce qu'exprimait déjà sur ce point Justus Möser (1842), p. 152: «Tant que nous ne hisserons pas le plan de notre histoire d'une manière ou d'une autre à un niveau d'unité, elle ressemblera toujours à un serpent qui, déchiqueté en cent morceaux, traîne avec lui [...] chaque partie de son corps ».

24. Sur la question des frontières de l'époque moderne: Lindgren (1997); Schmale (1998); Stauber (1998).

25. Voir récemment Edel (1997); Heil (1998).

26. Press (1970) ; Krüger (1980) ; Vann (1986) ; Weber (1998); Klinger (2002).

27. Schilling, H. (1988), (1991).

28. Reden-Dohna (1982) ; Schmidt, G. (1984), (1989) ; Schmidt, H. R. (1986) ; Böhme (1989) ; Arndt (1991) ; Press (1998a), (1998b).

29. Press (1986), (1989), (1991); Schindling / Ziegler (1990).

30. Aulinger (1980) ; Neuhaus (1982) ; Schindling (1991); Härter (1992).

31. Smend (1911) ; Press (1987) ; Diestelkamp (1990), (1993) ; Scheurmann (1994).

32. Schulze (1978); Schmid, P. (1983).

33. Hye (1973) ; Hattenhauer (1990), S. 95-105 ; Hess (1997) ; Kiessling (1997) ; Müller, R. A (1997), les autres contributions de ce recueil fournissent également une riche documentation iconographique. 
34. Krieger (1957) ; Willoweit (1984) ; Maier (1992) ; Schmidt, G. (1997), (2001b), (2001a).

35. Behringer (1990), (1998).

36. Une recherche fondamentale et synthétique sur les guerres que dut mener l'Empire à l'époque moderne reste encore à écrire. Müller, K. (1973) ; Neuhaus (1986) ; Burkhardt (1997) ; Schulze (1978). Sur la politique militaire des cercles du Sud et de l'Ouest de l'Empire, voir Plassmann (2000).

37. Pour une synthèse toujours actuelle : Heckel (1983), p. 33-66.

38. Dickmann (1998); Schmidt, G. (1998), (2001a).

39. C'est-à-dire la Prusse [NDT]. Voir Vann (1975) ; Gittel (1996) ; Hartmann (1997) ; Dotzauer (1998) ; Wüst (2000).

40. Neuhaus (1989) ; Aretin (1993-2000), ici : vol. 3, p. 87 sq. ; Externbrink (2001) ; Schmidt, G. (1999), p. 273 sq.

41. Jörn / North (2000).

42. Schulze (1975), (1980) ; Trossbach (1985) ; Blickle (1988) ; Schmidt, G. (1991) ; Gabel (1995).

43. Westphal (2002).

44. Kohler (1990); Schindling (1991); Lanzinner (1993); Härter (1993); Luttenberger (1994). Davantage critique : Aretin (1989); Duchhardt (1990), (1997).

45. Schulze (1978), p. 363.

46. Buschmann (1984), p. 219 sq. et 223. Sur les conceptions de la patrie appliquées à l'Allemagne à la fin du XVIe siècle, voir Schmidt, A. (2007).

47. Muhlack (1989); Wiegels / Woesler (1995); Wagner (1996); Münkler / Grünberger (1998).

48. Moraw (1988) ; Press (1988a) ; Aretin (1988). Sur les cercles matrimoniaux, voir par exemple Schmidt, G. (1989), p. 478-490.

49. Hammerstein (1972), (1985), (1995).

50. Voir Raumer (1952) ; Asbach (2001b), p. 1082 sq. Sur cette mésinterprétation, voir Burgdorf (1999), p. 49 sq.

51. Quelques exemples sont fournis par Schmidt, G. (2002), p. 29 sq.

52. Voir Schmidt, G. (1999), p. 92 sq.

53. Zwiedineck-Südenhorst (1888); Schilling, M. (1990); Bosbach (1992); Hollenbeck (1999); Schmidt, P. (2001).

54. Duggan (1989).

55. Biedermann (1883) ; Häpke (1926) ; Bog (1959).

56. Volckart (1999).

57. Sous cet angle significatif : Heeren (1819).

58. Ernstberger (1932).

59. Aretin (1993-2000), vol. 3, p. 468-512 ; Härter (1992).

60. Burg (1989).

61. Hegel (1998); Schiller (1983).

62. Meinecke (1908); voir également Kemiläinen (1956).

63. Schmidt, G. (2002).

64. Hegel (1998), p. 6 et passim.

65. Heller (1921).

66. Suivre ici Schmidt, G. (2002).

67. Voir, entre autres, Nipperdey (1983), p. 14. - La célèbre citation de Goethe sur la disparition du Saint-Empire dit même le contraire si l'on retient son contexte. Voir ici Schmidt, G. (1999), p. 346. Une étude récente suggestive : Mader (2001).

*. NDT : la traduction française de ces extraits du Discours à la nation allemande est donnée sur l'édition de 1992, Paris, Imprimerie Nationale, dans la traduction d'Alain Renault (prés., trad., comm.), ici pages 393 sq. L'autre traduction française de référence est celle parue en 1975, Paris, 
Aubier-Montaigne, par Sophie Jankélévitch avec une introduction de Max Rouché, qui remplace avantageusement celles de 1895 et de 1923.

68. Fichte (1971 [1846]), p. 259-499 : « Reden an die deutsche Nation », 8e et 9e discours, citations p. 392 sq. et 396 sq. ; trad. fr. : Fichte (1992), p. 393 sq. Voir Meinecke (1908), p. 105 sq.

69. Schräder (1996).

70. Fischer (2000), p. 65.

71. Wehler (1987), p. 506-530; Wehler (2001), p. 62-73; Whaley (1996).

72. Press (1988b) ; Aretin (1993-2000), vol. 3, p. 489-528 ; Schmidt, G. (1999), p. 342-346.

73. Bogdandy (1991), en particulier les pages 521 sq. ; Grassmann (2000) ; Weisser-Lohmann (2000). Sur Fichte, voir Fischer (2000).

74. Zimmer (1971) ; Kaiser (1973) ; Hoover (1986) ; Johnston (1990) ; Graf (2000) ; Wehler (2001), en particulier les pages 69 sq. ; Echternkamp (2001).

75. Schmidt, G. (2000).

76. Voir le mémoire adressé le 17 septembre 1812 par le baron Charles Henri de Stein au tsar Alexandre Ier, dans lequel il rappelle les "guerres civiques" de 1740 et 1756 et le déclin de l'Allemagne qu'elles ont préparées, et où il en appelle à la destruction de la Prusse pour " préserver de quelque manière l'unité ». Stein (1961), p. 742 sq., citations p. 743.

77. Langewiesche (2000a), (2000b).

78. Angermeier (1991).

79. Schmidt, G. (1993), (1995); Schuck (1994); Billinger Jr. (1998).

80. Müller, H. (1989); Siemann (1995), p. 320-330.

81. A partir du projet d'un traité politique datant du printemps 1813, in : Fichte (1971 [1846]), p. 546-573, citations p. 554 et 565. Vgl. Meinecke (1908), p. 110 ; Winkler (2000), vol. 1, p. 60.

82. Wehler (1987), p. 528.

83. Steiger (1991) ; Hardtwig (1994); Bauer (1997).

84. Echternkamp (1998); Jeismann (1992), en particulier les p. 27-76.

85. Hansemann (1919). Cité d'après Echternkamp (1998), p. 73 ; Müller, H. (1989), p. 66.

86. Stenzel (1837), p. 8 sq.

87. Johann Friedrich Böhmer, fragment intitulé "Das Reich und die Stämme ", cité d'après Brechenmacher (1996), p. 148.

88. Droysen (1868), p. 4: «La Prusse aussi n'incorpore que des fragments du peuple et du pays allemands. Mais pour la création et la conservation de cet État, chaque fonction participe de l'ensemble, dont il a aggloméré pied à pied d'autres parties ».

89. Schmidt, H. (1988). Voir Langewiesche, «Reich, Nation und Staat in der jüngeren deutschen Geschichte », in : Langewiesche (2000b), p. 190-216, ici p. 204 sq.

90. Sur la postérité de ce modèle historiographique : Althoff (2000).

91. Voir Treitschke (1882).

92. Voir Brechenmacher (1996).

93. Müller, H. (1989), p. 68 sq.

94. Treitschke (1882), vol. 1 ; Schönemann (1989). Voir également la contribution de Wolgast (2002).

95. Cité d'après Biefang (1996), p. 401.

96. Meinecke (1908), p. 33

97. Meinecke (1908), p. 319 sq.

*. NDT : l'expression allemande «Krähwinkel », utilisée pour la première fois par Jean Paul en 1801 puis par Kotzebue dans sa pièce satirique «Die deutschen Kleinstädter » en 1803, censée renvoyer à un lieu fictif en Prusse, a très vite désigné un «bled » de province dans lequel rien ne se passe, puis ses habitants, puis des soldats bons à rien que l'on ne mobilise que quand plus aucune autre troupe ne peut être formée, pour finir par qualifier un événement sans importance. 98. Straub (2001), p. 15. 
99. Press (1989), p. 51. Sur les jugements portés récemment sur l'Empire, voir aussi : Schindling (2001).

100. Voir, entre autres Immich (1905), p. 34 sq. L'exemple de Ludwig Dehio montre combien cette perspective prussienne a encore pu encombrer l'historiographie allemande d'après 1945 : «Et même s'il nous faut bien prendre acte, dans ce monde bouleversé, de la fin de la ligne prussienne dans notre histoire, rien ne nous pousse pour autant, en dépit du cruel final, à nous en séparer en en diffamant le cours général! Ne nous serait-il pas possible de placer la mémoire de Frédéric et de Bismarck dans la vitrine de nos souvenirs, comme la précieuse porcelaine de nos aïeux que l'on ne met plus sur la table [...]» (Dehio [1961], p. 90).

101. Press (1989), p. 52.

102. Lutz (1958), (1964).

103. Consulter ses recueils d'articles : Oestreich (1969), (1980).

104. Schubert (1966).

105. Aretin (1967).

106. Caractéristique d'une tendance à la réévaluation du Saint-Empire : Buchheim (1961).

*. NDT : le sens de «systematisch» dans l'original allemand ne peut se comprendre que rapporté aux discussions consacrées aux tentatives de répartition au sein des facultés de sciences sociales et humaines entre des disciplines opérant par normes et «systèmes » et réputées de ce fait plus proches des sciences naturelles et des disciplines plus « historiques » et empiriques.

107. Press (1997), p. 18-41.

108. Voir Sailer (1999). Voir aussi Zorn (1959).

109. Reinhard (1999), p. 48.

110. Burkhardt (1999), p. 752.

111. Sur ce point, Burgdorf (2001a). Voir aussi Schrader (2001), en particulier les p. 112 sq.

112. Vgl. Neuhaus (1997).

113. Schmidt, G. (1999), (1996).

114. L'abbé de Saint Pierre a voulu voir dans le maintien des structures impériales et dans la concurrence entre un Empire de nature monarchique d'une part et de nature fédérative et confédérale de l'autre la faute originelle de ce qu'il désignait par ailleurs comme une fédération quasi idéale d'États et élevait au rang de modèle exemplaire pour l'Europe. Voir Asbach (2001a), en particulier les p. 210 sq.

115. Schilling, H. (2001), p. 393.

116. Winkler (2000), vol. 1, p. 5.

117. Passage cité en anglais dans le texte [NDT]. Whaley (2002), p. 28.

118. Schorn-Schütte (1993) ; van Gelderen (1996b) ; Friedeburg (2000).

119. Voir avant tout Schmidt, H. R. (1997).

120. Croxton (1999), p. 570.

121. Voir Ogilvie (1999); Neugebauer (2001).

*. En anglais dans le texte [NDT].

122. Beik (1985) ; Croxton (1999), p. 583.

123. "Historische Wege nach Europa », in : Langewiesche (2000b), p. 217-230.

124. Voir Huhn (1990), p. 56 sq., p. 60 et 62 puis suiv. Citation d'après Whaley (2002), p. 22 sq.

125. Gaupp (1948), p. 3-7.

126. Voir l'article de la Frankfurter Allgemeine Zeitung du 31.05.2000, N 5.

127. Schilling, H. (2001), p. 394.

128. Reinhard (2001).

129. Weisert (1994).

130. Contrairement à Dann (2001), p. 266.

131. Voir Schnettger (2002).

*. NDT : en français dans le texte. 
132. Citation donnée en anglais dans le texte [NDT]. Whaley (2002), p. 35.

INDEX

Schlüsselwörter : Altes Reich, Historiographie, politische Geschichte der Frühen Neuzeit, Verfassungsgeschichte

Mots-clés : histoire politique moderne, historiographie, institutions, Saint-Empire

\section{AUTEURS}

\section{GEORG SCHMIDT}

Georg Schmidt est professeur d'histoire moderne à l'Université de Iéna. Pour plus d'informations, voir la notice suivante. 